\title{
PRICING EQUITY DERIVATIVES SUBJECT TO BANKRUPTCY
}

\author{
VADIM LINETSKY \\ Northwestern University, Evanston, Illinois
}

\begin{abstract}
We solve in closed form a parsimonious extension of the Black-Scholes-Merton model with bankruptcy where the hazard rate of bankruptcy is a negative power of the stock price. Combining a scale change and a measure change, the model dynamics is reduced to a linear stochastic differential equation whose solution is a diffusion process that plays a central role in the pricing of Asian options. The solution is in the form of a spectral expansion associated with the diffusion infinitesimal generator. The latter is closely related to the Schrödinger operator with Morse potential. Pricing formulas for both corporate bonds and stock options are obtained in closed form. Term credit spreads on corporate bonds and implied volatility skews of stock options are closely linked in this model, with parameters of the hazard rate specification controlling both the shape of the term structure of credit spreads and the slope of the implied volatility skew. Our analytical formulas are easy to implement and should prove useful to researchers and practitioners in corporate debt and equity derivatives markets.
\end{abstract}

KEY WORDS: bankruptcy, credit risk, hazard rate, credit spread, stock options, implied volatility skew, Asian options, Brownian exponential functionals, Schrödinger operator with Morse potential, spectral expansions

\section{INTRODUCTION}

In the celebrated Black-Scholes-Merton model the firm's stock price is assumed to follow geometric Brownian motion (GBM) - a diffusion process with constant volatility and infinite life time. On one hand, this assumption precludes bankruptcy. To the contrary, modeling bankruptcy and credit spreads is at the center of the literature on corporate bonds (see recent monographs Bielecki and Rutkowski [2002], Duffie and Singleton [2003], Lando [2004], and Schönbucher [2003], for a summary of the credit risk literature). On the other hand, the GBM assumption contradicts the accumulated empirical evidence on implied volatility skews exhibited by stock options prices. Modeling the implied volatility skew is at the center of the equity derivatives literature (e.g., Rubinstein 1994; Jackwerth and Rubinstein 1996).

Until recently, the literature on stock options and the literature on corporate bonds developed more or less independently. Recently, the two strands of literature have merged on the topic of modeling convertible bonds, as convertible bonds are corporate bonds with embedded stock option features. In the reduced-form framework, one specifies the hazard rate of bankruptcy as a decreasing function of the underlying stock price, $h=h(S)$. The bankruptcy event is modeled as the first jump time of a doubly stochastic Poisson

This research was supported by the U.S. National Science Foundation under Grant DMI-0200429.

Manuscript received October 2004; final revision received January 2005.

Address correspondence to V. Linetsky, Department of Industrial Engineering and Management Sciences, McCormick School of Engineering and Applied Sciences, Northwestern University, 2145 Sheridan Road, Evanston, IL 60208; e-mail: linetsky@iems.northwestern.edu. 
process with intensity $h$, and the term structure of credit spreads is determined from the specification of the intensity and the parameters of the underlying stock price process. The negative power intensity provides a particularly parsimonious specification:

$$
h(S)=\alpha S^{-p}
$$

for some $p>0$ and $\alpha>0$. This specification was recently employed in Andersen and Buffum (2003), Ayache, Forsyth, and Vetzal (2003), Davis and Lischka (2002), Duffie and Singleton (2003, p. 216), Muromachi (1999), and Takahashi, Kobayashi, and Nakagawa (2001) in the context of modeling convertible bonds. Muromachi (1999) empirically estimated the value of the power parameter $p$ to be in the range between 1.2 and 2 for Japanese bonds rated $\mathrm{BB}+$ and below. In these references the convertible bond price was determined numerically by finite-difference or lattice methods. The negative power intensity model has become popular among practitioners for convertible bond modeling. While the main focus of these references is on pricing convertible bonds, Andersen and Buffum (2003) showed that this class of models exhibits implied volatility skews in stock option prices, with the parameters of the hazard rate specification controlling the slope of the skew, thus establishing a link between implied volatility skews and credit spreads (see also Hull, Nelken, and White [2004] for the linkage between credit spreads and implied volatility skews in Merton's structural model).

In the present paper we solve the negative power intensity model in closed form both for corporate bonds and European-style stock options. We start in Section 2 by introducing bankruptcy into the GBM model by killing the process at the rate $h=h(S)$, where $h$ is a decreasing function of the underlying stock price. To insure that the discounted stock price is a martingale under equivalent martingale measure (EMM), the hazard rate $h(S)$ needs to be added to the drift rate of the process (e.g., Davis and Lischka 2002). We then discuss the pricing of equity derivatives in this diffusion-with-killing model and show that, by Girsanov's theorem, the risky discount factor with the hazard rate can be removed from the valuation relationships at the expense of modifying the drift of the underlying diffusion, thus reducing the problem to the study of the diffusion process (2.3) without killing. In Section 3 we adopt the negative power specification (1.1) and show that the valuation problem further reduces to the problem of computing expectations with respect to the distribution of the diffusion process $X^{(v)}$ solving the linear stochastic differential equation (SDE) (3.4). This process has recently been studied in the context of pricing arithmetic Asian options (see Donati-Martin, Ghomrasni, and Yor [2001] and Linetsky [2004a] and references therein). Fortunately, the spectral representation of its transition density is available in closed form (Proposition 3.3), and we are able to compute all the necessary integrals in closed form and obtain closed-form expressions for both corporate bonds (Proposition 3.4) and European-style stock options (Proposition 3.5). These closed-form expressions are in the form of spectral expansions (for recent applications of the spectral expansion method to derivatives pricing see Lewis [1998], Gorovoi and Linetsky [2004], Linetsky [2004a-2004d] and references therein). In Section 4 our analytical solutions are employed to develop an economic analysis of the model. In particular, we study possible shapes and asymptotics of the term structure of credit spreads and implied volatility skews in the negative power intensity model, and explore the link between credit spreads and implied volatility skews. Section 5 concludes the paper. Appendix A presents additional mathematical details on the process $X^{(v)}$ and its density $p^{(v)}$ and their connections with several classical mathematical objects (Schrödinger operator with Morse potential and Maass Laplacian on the hyperbolic plane). Appendix B discusses the close relationship of the process $X^{(v)}$ to the pricing of Asian options (Geman and Yor 1992, 1993; Donati-Martin, Ghomrasni, and Yor 2001; Linetsky 2004a). Appendix C collects 
the necessary facts about special functions appearing in the pricing formulas. Appendix D contains proofs.

\section{EQUITY DERIVATIVES SUBJECT TO BANKRUPTCY IN THE INTENSITY-BASED FRAMEWORK}

\subsection{The Stock Price Model}

Let $(\Omega, \mathcal{G}, \mathbb{P})$ be a probability space supporting a standard Brownian motion $\left\{B_{t}, t \geq\right.$ $0\}$ and an exponential random variable $e \sim \operatorname{Exp}(1)$ with unit parameter independent of $B$. Let $h=h(x)$ be a nonnegative function on $\mathbb{R}^{+}$satisfying the following assumptions.

Assumption 2.1. We assume that $h$ is $C^{1}(0, \infty)$, strictly decreasing, and has the following limits:

$$
\lim _{x \rightarrow 0} h(x)=+\infty, \quad \lim _{x \rightarrow \infty} h(x)=0 .
$$

We take an EMM $\mathbb{P}$ as given and model the pre-bankruptcy underlying stock price dynamics under the EMM as a diffusion process $\left\{S_{t}, t \geq 0\right\}$ solving the SDE

$$
d S_{t}=\left(r-q+h\left(S_{t}\right)\right) S_{t} d t+\sigma S_{t} d B_{t}, \quad S_{0}=S>0,
$$

where $r>0, q>0$, and $\sigma>0$ are the risk-free interest rate, dividend yield, and prebankruptcy stock price volatility, respectively. Under Assumption 2.1, the SDE (2.1) has a unique strong nonexploding solution. This solution is a diffusion process on $(0$, $\infty)$ with both zero and infinity inaccessible boundaries. To see this, consider a process $\left\{X_{t}:=\sigma^{-1} \ln S_{t}, t \geq 0\right\}$,

$$
d X_{t}=\left(\mu+\sigma^{-1} h\left(e^{\sigma X_{t}}\right)\right) d t+d B_{t}, \quad X_{0}=\sigma^{-1} \ln S, \quad \mu=\left(r-q-\sigma^{2} / 2\right) / \sigma .
$$

Under Assumption 2.1, this SDE has a unique strong nonexploding solution. Strong uniqueness up to the explosion time is insured by $h \in C^{1}$ (e.g., Ikeda and Watanabe 1981). Nonexplosion can be checked by applying Feller's test for explosions (e.g., Karlin and Taylor 1981, p. 234).

We model the random time of bankruptcy $\tau$ as the first time when the process $\int_{0}^{t} h\left(S_{u}\right) d u$ is greater or equal to the random level $e$ (equivalently, as the first jump time of a doubly stochastic Poisson process (Cox process) with intensity (hazard rate) $h_{t}=h\left(S_{t}\right)$ ):

$$
\tau=\inf \left\{t \geq 0: \int_{0}^{t} h\left(S_{u}\right) d u \geq e\right\} .
$$

At the time of bankruptcy $\tau$, the stock price jumps to the bankruptcy state, $\Delta$, where it remains forever ( $\Delta$ is a cemetery state in the terminology of Markov processes; e.g., Revuz and Yor 1999). We assume that equity holders do not receive any recovery in the event of bankruptcy and their equity position becomes worthless. In other words, we model the stock price subject to bankruptcy as a diffusion process $\left\{S_{t}^{\Delta}, t \geq 0\right\}$ with the extended state space $E^{\Delta}=(0, \infty) \cup\{\Delta\}$, diffusion coefficient $a(x)=\sigma x$, drift $b(x)=(r-q+h(x)) x$, and killing rate $h(x)$. In our notation, $\left\{S_{t}, t \geq 0\right\}$ is the pre-bankruptcy stock price process (2.1), while $\left\{S_{t}^{\Delta}, t \geq 0\right\}$ is the stock price process subject to bankruptcy, so that $S_{t}^{\Delta}=S_{t}$ for $t<\tau$ and $S_{t}^{\Delta}=\Delta$ for all $t \geq \tau$.

The addition of the hazard rate in the drift rate in the pre-bankruptcy dynamics (2.1) compensates for the bankruptcy jump to insure that the total expected rate of return to the 
stockholder is equal to the risk-free rate in the risk-neutral economy and the discounted gain process is a martingale under EMM (e.g., Davis and Lischka 2002). Our assumptions about the hazard rate are intuitive. As the stock price declines towards zero, the hazard rate increases to infinity. As the stock price increases, the hazard rate declines to zero, making the stock price process asymptotically GBM.

To keep track of how information is revealed over time, following Elliot et al. (2000), we introduce a bankruptcy jump indicator process $\left\{D_{t}, t \geq 0\right\}, D_{t}=1_{\{t \geq \tau\}}$, denote by $\mathbb{D}=\left\{\mathcal{D}_{t}, t \geq 0\right\}$ a filtration generated by $D$, by $\mathbb{F}=\left\{\mathcal{F}_{t}, t \geq 0\right\}$ a filtration generated by the Brownian motion $B$, and by $\mathbb{G}=\left\{\mathcal{G}_{t}, t \geq 0\right\}, \mathcal{G}_{t}=\mathcal{F}_{t} \vee \mathcal{D}_{t}$, an enlarged filtration. The defaultable stock process $S^{\Delta}$ is adapted to the enlarged filtration $\mathbb{G}$.

If we identify the cemetery state $\Delta=0$, then we can write the process for the stock price subject to bankruptcy in the form

$$
d S_{t}^{\Delta}=S_{t-}^{\Delta}\left((r-q) d t+\sigma d B_{t}-d M_{t}\right)
$$

where

$$
M_{t}=D_{t}-\int_{0}^{t \wedge \tau} h\left(S_{u}\right) d u,
$$

is a martingale (compensated bankruptcy jump process).

\subsection{Equity Derivatives}

A European-style equity derivative with maturity (expiration) at time $T>0$ is defined by its payoff $F: E^{\Delta} \rightarrow \mathbb{R}^{+}$,

$$
F\left(S_{T}^{\Delta}\right)=F\left(S_{T}^{\Delta}\right) \mathbf{1}_{\left\{S_{T}^{\Delta} \neq \Delta\right\}}+R \mathbf{1}_{\left\{S_{T}^{\Delta}=\Delta\right\}},
$$

where $F\left(S_{T}^{\Delta}\right) \mathbf{1}_{\left\{S_{T}^{\Delta} \neq \Delta\right\}}$ is the payoff at maturity, given no bankruptcy prior to $T$, and $R=$ $F(\Delta) \geq 0$ is the recovery payment at maturity in the event of bankruptcy. The valuation of the two parts of the payoff is standard in the reduced-form intensity-based credit risk modeling framework ${ }^{1}$

$$
\begin{aligned}
& e^{-r T} \mathbb{E}\left[F\left(S_{T}^{\Delta}\right) \mathbf{1}_{\left\{S_{T}^{\Delta} \neq \Delta\right\}}\right]=e^{-r T} \mathbb{E}\left[F\left(S_{T}\right) \mathbf{1}_{\{\tau>T\}}\right] \\
& \quad=e^{-r T} \mathbb{E}\left[F\left(S_{T}\right) \mathbb{E}\left[\mathbf{1}_{\{\tau>T\}} \mid \mathcal{F}_{T}\right]\right]=e^{-r T} \mathbb{E}\left[e^{-\int_{0}^{T} h\left(S_{t}\right) d t} F\left(S_{T}\right)\right],
\end{aligned}
$$

and

$$
\begin{aligned}
& e^{-r T} \mathbb{E}\left[R \mathbf{1}_{\left\{S_{T}^{\Delta}=\Delta\right\}}\right]=e^{-r T} \mathbb{E}\left[R \mathbf{1}_{\{\tau \leq T\}}\right]=e^{-r T} R\left(1-\mathbb{E}\left[\mathbf{1}_{\{\tau>T\}}\right]\right) \\
& \quad=e^{-r T} R\left(1-\mathbb{E}\left[\mathbb{E}\left[\mathbf{1}_{\{\tau>T\}} \mid \mathcal{F}_{T}\right]\right]\right)=e^{-r T} R\left(1-\mathbb{E}\left[e^{-\int_{0}^{T} h\left(S_{t}\right) d t}\right]\right),
\end{aligned}
$$

where $\left\{\mathcal{F}_{t}, t \geq 0\right\}$ is the filtration generated by the Brownian motion $B$, and we used the fact that

$$
\mathbb{E}\left[\mathbf{1}_{\{\tau>T\}} \mid \mathcal{F}_{T}\right]=e^{-\int_{0}^{T} h\left(S_{t}\right) d t} .
$$

These valuations reduce to computing expectations of the form

$$
V_{\psi}(S, T)=e^{-r T} \mathbb{E}\left[e^{-\int_{0}^{T} h\left(S_{t}\right) d t} \psi\left(S_{T}\right)\right] .
$$

\footnotetext{
${ }^{1}$ See Duffie, Schroder, and Skiadas (1996), Duffie and Singleton (1999), Jarrow and Turnbull (1995), Jarrow, Lando, and Turnbull (1997), and Madan and Unal (1998), as well as recent monographs Bielecki and Rutkowski (2002), Duffie and Singleton (2003), Lando (2004), and Schönbucher (2003). For the partial differential equation (PDE) approach to the pricing of equity derivatives with the hazard rate dependent on the underlying stock price, see Carr and Javaheri (2005).
} 
This can be interpreted as pricing a claim with payoff $\psi\left(S_{T}\right)$ at $T>0$ in a fictitious economy with the risk-free interest rate process $r_{t}=r+h\left(S_{t}\right)$, stock price process (2.1), and no bankruptcy. The discount factor with $h$ can be removed by changing the probability measure.

Proposition 2.1.

$$
V_{\psi}(S, T)=e^{-r T} \mathbb{E}\left[e^{-\int_{0}^{T} h\left(S_{t}\right) d t} \psi\left(S_{T}\right)\right]=e^{-q T} S \hat{\mathbb{E}}\left[S_{T}^{-1} \psi\left(S_{T}\right)\right],
$$

where $\hat{\mathbb{E}}$ is the expectation with respect to the probability measure $\hat{\mathbb{P}}$ under which $\hat{B}_{t}:=$ $B_{t}-\sigma t$ is a standard Brownian motion and

$$
d S_{t}=\left(r-q+\sigma^{2}+h\left(S_{t}\right)\right) S_{t} d t+\sigma S_{t} d \hat{B}_{t}, \quad S_{0}=S>0 .
$$

Proof. From equation (2.1) we have

$$
S_{t}=S e^{(r-q) t+\int_{0}^{t} h\left(S_{u}\right) d u+\sigma B_{t}-\frac{1}{2} \sigma^{2} t}, \quad t \geq 0,
$$

and hence

$$
e^{-r T} \mathbb{E}\left[e^{-\int_{0}^{T} h\left(S_{t}\right) d t} \psi\left(S_{T}\right)\right]=e^{-q T} S \mathbb{E}\left[e^{\sigma B_{T}-\frac{1}{2} \sigma^{2} T} S_{T}^{-1} \psi\left(S_{T}\right)\right] .
$$

Application of Girsanov's theorem completes the proof.

In particular, for fixed $T>0$, we will be interested in a zero-coupon bond with unit face value and constant recovery payment $0 \leq R<1$ at maturity, a call option with strike $K>0$ with the payoff $\left(S_{T}-K\right)^{+}$at expiration and no recovery if the firm goes bankrupt, and a put option with strike $K>0$ with the payoff $\left(K-S_{T}\right)^{+}$if $\tau>T$ and recovery payment $R=K$ at expiration in the event of bankruptcy $\tau \leq T$ (note that we decompose the put payoff into two parts: $\left.\left(K-S_{T}\right)^{+} \mathbf{1}_{\{\tau>T\}}+K \mathbf{1}_{\{\tau \leq T\}}\right)$. Using Proposition 2.1, the pricing formulas for the bond, call, and put take the form

$$
B_{R}(S, T)=e^{-r T} R+(1-R) e^{-q T} S \hat{\mathbb{E}}\left[S_{T}^{-1}\right]
$$

respectively. In particular, the put-call parity is satisfied by

$$
C_{K}(S, T)-P_{K}(S, T)=e^{-q T} S-e^{-r T} K
$$

One notes that the put pricing formula (2.4c) consists of two parts: the present value of the put payoff given no bankruptcy,

$$
e^{-q T} S \hat{\mathbb{E}}\left[\left(K S_{T}^{-1}-1\right)^{+}\right],
$$

and the present value of the recovery in the event of bankruptcy,

$$
K\left(e^{-r T}-B(S, T)\right)
$$

where

$$
B(S, T)=e^{-r T} \mathbb{P}(\tau>T)=e^{-q T} S \hat{\mathbb{E}}\left[S_{T}^{-1}\right]
$$


is the price of the $T$-maturity zero-coupon bond with unit face value and zero recovery (the subscript 0 in $B_{0}(S, T)$ will be dropped for zero-recovery bonds) and $\mathbb{P}(\tau>T)$ is the (risk-neutral) probability of surviving beyond time $\mathrm{T}$ (survival probability). The recovery part of the put option is termed a bankruptcy claim by Lewis (1998) in the context of Merton's model with constant dividend rate, where the bankruptcy occurs when the stock price process hits zero.

\section{ANALYTICAL SOLUTION FOR THE NEGATIVE POWER INTENSITY MODEL}

\subsection{The Negative Power Intensity Model}

A parsimonious specification for the hazard rate satisfying Assumption 2.1 is

$$
h(S)=\alpha S^{-p}, \quad \alpha>0, \quad p>0 .
$$

This specification was employed in Andersen and Buffum (2003), Ayache, Forsyth, and Vetzal (2003), Davis and Lischka (2002), Duffie and Singleton (2003, p. 216), Muromachi (1999), and Takahashi, Kobayashi, and Nakagawa (2001) in the context of modeling convertible bonds. Muromachi (1999) estimated the value of the power parameter $p$ to be in the range between 1.2 and 2 for Japanese bonds rated $\mathrm{BB}+$ and below. In these references the convertible bond price was determined numerically by finite-difference or lattice methods. The negative power intensity model (3.1) has become popular among practitioners for convertible bond modeling. In the present paper we solve the model both for corporate bonds and European-style stock options in closed form.

We are interested in calculating expectations (2.2) under the process (2.3) with $h$ given by Itô's (3.1). First, introduce a new process $\left\{Z_{t}:=\beta S_{t}^{p}, t \geq 0\right\}$, where $\beta=p \sigma^{2} /(4 \alpha)$. From Itô's formula, it solves the linear SDE

$$
d Z_{t}=\left(a Z_{t}+b\right) d t+c Z_{t} d B_{t}, \quad Z_{0}=z=\beta S^{p},
$$

with parameters

$$
a=p\left(r-q+(p+1) \sigma^{2} / 2\right) \in \mathbb{R}, \quad b=p^{2} \sigma^{2} / 4>0, \quad c=p \sigma>0 .
$$

This linear SDE has a well-known solution (e.g., Karatzas and Shreve 1992, pp. 360-361)

$$
Z_{t}=e^{\left(a-c^{2} / 2\right) t+c B_{t}}\left(z+b \int_{0}^{t} e^{-\left(a-c^{2} / 2\right) u-c B_{u}} d u\right), \quad t \geq 0 .
$$

Using the Brownian scaling property $c B_{t} \stackrel{\text { (law) }}{=} 2 B_{c^{2} t / 4}$, we can effect a time change so that

$$
Z_{t}=X_{\tau(t)}^{(v)}, \quad \tau(t)=p^{2} \sigma^{2} t / 4,
$$

where $X^{(v)}$ is the standardized process:

$$
X_{t}^{(v)}=e^{2\left(v t+B_{t}\right)}\left(z+\int_{0}^{t} e^{-2\left(v u+B_{u}\right)} d u\right), \quad t \geq 0, \quad \text { with } \quad v=\frac{2}{p \sigma^{2}}\left(r-q+\frac{\sigma^{2}}{2}\right),
$$

solving the standardized linear $\mathrm{SDE}^{2}$

$$
d X_{t}^{(v)}=\left(2(v+1) X_{t}^{(v)}+1\right) d t+2 X_{t}^{(v)} d B_{t}, \quad X_{0}^{(v)}=x=z=\beta S^{p} .
$$

\footnotetext{
${ }^{2}$ We note that this SDE was derived by Shiryaev (1961) in the context of quickest detection problems (see Peskir 2004).
} 
Thus, the valuation equation (2.2) is reduced to

$$
V_{\psi}(S, T)=e^{-q T} S E_{x}^{(v)}\left[\chi_{\psi}\left(X_{\tau}^{(v)}\right)\right], \quad x=\beta S^{p}, \quad \beta=p \sigma^{2} /(4 \alpha), \quad \tau=p^{2} \sigma^{2} T / 4,
$$

where the expectation $E_{x}^{(v)}$ is with respect to the law of the process $X^{(v)}$ started at $x$ and

$$
\chi_{\psi}(y):=(y / \beta)^{-1 / p} \psi\left((y / \beta)^{1 / p}\right)
$$

\subsection{The Process $X^{(v)}$, Its Resolvent Kernel, and Transition Density}

In this section we review the properties of the diffusion process $X^{(v)}$.

Proposition 3.1. The boundary at zero is entrance for all $v \in \mathbb{R}$. The boundary at infinity is natural for all $v \in \mathbb{R}$. For $v>0$ it is attracting and the process is transient. For $v \leq 0$ it is nonattracting. For $v<0$ the process is positive recurrent and possesses a stationary distribution with the density

$$
\pi(x)=\frac{2^{v}}{\Gamma(-v)} x^{\nu-1} e^{-\frac{1}{2 x}}
$$

Proof. The scale and speed densities of $X^{(v)}$ are (see, e.g., Borodin and Salminen 2002, p. 17, for scale and speed densities of one-dimensional diffusions)

$$
\mathfrak{s}(x)=x^{-\nu-1} e^{\frac{1}{2 x}}, \quad \mathfrak{m}(x)=\frac{1}{2} x^{\nu-1} e^{-\frac{1}{2 x}} .
$$

The nature of the boundaries at zero and infinity are established by applying Feller's boundary classification criteria based on the behavior of the scale and speed densities (Borodin and Salminen 2002, pp. 14-15). For $v<0$ the speed measure is finite, $\int_{0}^{\infty} m(x) d x<\infty$, and hence, the process is positive recurrent with the stationary density given by the normalized speed density (Borodin and Salminen 2002, pp. 20-21).

Let $p^{(v)}(t ; x, y)$ be the transition density of $X^{(v)}$ and, for $s>0, G_{s}^{(v)}(x, y)$ its Laplace transform in time (also called resolvent kernel or Green's function, e.g., Borodin and Salminen 2002, pp. 19-20) ${ }^{3}$

$$
G_{s}^{(v)}(x, y)=\int_{0}^{\infty} e^{-s t} p^{(v)}(t ; x, y) d t
$$

Proposition 3.2. For $x, y>0$, the resolvent kernel is given by $(x \wedge y:=\min \{x, y\}$, $x \vee y:=\max \{x, y\})$ :

$$
G_{s}^{(v)}(x, y)=\Gamma\left(\mu(s)+\frac{v}{2}\right)\left(\frac{y}{x}\right)^{\frac{v-1}{2}} e^{\frac{1}{4 x}-\frac{1}{4 y}} \mathcal{M}_{\frac{1-v}{2}, \mu(s)}\left(\frac{1}{2(x \vee y)}\right) W_{\frac{1-v}{2}, \mu(s)}\left(\frac{1}{2(x \wedge y)}\right),
$$

where

$$
\mu(s)=\frac{1}{2} \sqrt{2 s+v^{2}}
$$

\footnotetext{
${ }^{3}$ Note that our Green's function and transition density are defined with respect to the Lebesgue measure, while Borodin and Salminen's are defined with respect to the speed measure and, thus, our Green's function and transition density differ from Borodin and Salminen's by a factor of $\mathfrak{m}(y)$.
} 
$\Gamma(z)$ is the Gamma function, and $\mathcal{M}_{\kappa, \mu}(z)$ and $W_{\kappa, \mu}(z)$ are the Whittaker functions (C.2) and (C.3). For $x=0$ and $y>0$ the Green's function reduces to

$$
G_{s}^{(v)}(0, y)=\Gamma\left(\mu(s)+\frac{v}{2}\right)(2 y)^{\frac{v-1}{2}} e^{-\frac{1}{4 y}} \mathcal{M}_{\frac{1-v}{2}, \mu(s)}\left(\frac{1}{2 y}\right) .
$$

Proof. See Appendix D.

REMARK 3.1. This Green's function was obtained by Donati-Martin, Ghomrasni, and Yor (2001, Theorem 3.1) by solving the differential equation. The transition density is recovered by inverting the Laplace transform. This produces the spectral representation for the transition density (see McKean [1956] and Itô and McKean [1974, Section 4.11] for the spectral representation of the transition density of a one-dimensional diffusion, and Linetsky [2004b; 2006] and references therein for applications in finance).

Proposition 3.3. For $x, y>0$ and $v \in \mathbb{R}$, the transition density has the following spectral representation

$$
\begin{aligned}
p^{(v)}(t ; x, y)= & \mathbf{1}_{\{v<0\}} \pi(y)+\mathbf{1}_{\{v<-2\}} \sum_{n=1}^{[|v| / 2]} e^{-2 n(|v|-n) t} \frac{2(|v|-2 n) n !}{\Gamma(1+|\nu|-n)} e^{-\frac{1}{2 y}}(2 x)^{n}(2 y)^{n-1-|v|} \\
& \times L_{n}^{(|v|-2 n)}\left(\frac{1}{2 x}\right) L_{n}^{(|v|-2 n)}\left(\frac{1}{2 y}\right)+\frac{1}{2 \pi^{2}} \int_{0}^{\infty} e^{-\frac{\left(\nu^{2}+\rho^{2}\right) t}{2}} e^{\frac{1}{4 x}-\frac{1}{4 y}}\left(\frac{y}{x}\right)^{\frac{v-1}{2}} \\
& \times W_{\frac{1-v}{2}, \frac{i \rho}{2}}\left(\frac{1}{2 x}\right) W_{\frac{1-v}{2}, \frac{i \rho}{2}}\left(\frac{1}{2 y}\right)\left|\Gamma\left(\frac{v+i \rho}{2}\right)\right|^{2} \sinh (\pi \rho) \rho d \rho,
\end{aligned}
$$

where $L_{n}^{(\alpha)}(x)$ are the generalized Laguerre polynomials, $[x]$ denotes the integer part of $x$, and $\mathbf{1}_{\{\cdot\}}$ is the indicator. When $x=0, y>0$, and $v \in \mathbb{R}$,

$$
\begin{aligned}
p^{(v)}(t ; 0, y)= & \frac{1}{2 \pi^{2}} \int_{0}^{\infty} e^{-\frac{\left(\nu^{2}+\rho^{2}\right) t}{2}} e^{-\frac{1}{4 y}}(2 y)^{\frac{v-1}{2}} W_{\frac{1-v}{2}, \frac{i \rho}{2}}\left(\frac{1}{2 y}\right)\left|\Gamma\left(\frac{v+i \rho}{2}\right)\right|^{2} \sinh (\pi \rho) \rho d \rho \\
& +\mathbf{1}_{\{v<0\}} \pi(y)+\mathbf{1}_{\{v<-2\}} \sum_{n=1}^{[|v| / 2]} e^{-2 n(|v|-n) t} \frac{(-1)^{n} 2(|v|-2 n)}{\Gamma(1+|v|-n)} \\
& \times e^{-\frac{1}{2 y}}(2 y)^{n-1-|v|} L_{n}^{(|v|-2 n)}\left(\frac{1}{2 y}\right) .
\end{aligned}
$$

Proof. See Appendix D.

REMARK 3.2. The density in Proposition 3.3 has a long history and is closely related to a number of classical mathematical objects. For $v<0$ this density was first obtained by Wong (1964, p. 271, equation (38)) in his studies of diffusions with stationary densities in the Pearson family. See Comtet, Monthus, and Yor (1998), Linetsky (2004a), and Appendix A and B for details. In Appendix D we provide a proof for all $v \in \mathbb{R}$.

REMARK 3.3. Because the boundary at zero is entrance, the process can be started at zero and $G_{s}^{(v)}(0, y)$ and $p^{(v)}(t ; 0, y)$ exist. In the application to Asian options the process is started at zero (see Appendix B). In the present application to equity derivatives subject to bankruptcy, the process is started at a positive value $x=\beta S^{p}>0$. 
REMARK 3.4. For $v \geq 0$ the spectrum of the infinitesimal generator

$$
\mathcal{G}^{(v)}=2 x^{2} \frac{d^{2}}{d x^{2}}+(2(v+1)+1) \frac{d}{d x}
$$

of $X^{(v)}$ in the Hilbert space of functions square-integrable with the speed density $\mathfrak{m}$ in equation (3.8) is purely continuous (the integral term in the spectral expansion (3.12)). For $v<0$ there is some nonempty discrete spectrum that contains at least the zero principal eigenvalue. The term in the spectral expansion (3.12) corresponding to the zero principal eigenvalue is the stationary density $\pi$ (3.7). The spectral expansion for the transition density can be obtained by directly inverting the Laplace transform of the resolvent kernel, using the Cauchy Residue Theorem. The resolvent kernel (3.10) needs to be considered in the complex $s$-plane. The poles of the resolvent kernel (the poles of the Gamma function in equation $(3.10)$ for $v<0$ ) give the eigenvalues, and the integral along the branch cut $\left\{s=-v^{2} / 2-\rho^{2} / 2, \rho \in[0, \infty)\right\}$ produces the continuous part of the spectral expansion. See Appendix D for this approach. Alternatively, the spectral expansion can be obtained by employing the real-variable approach by first considering a truncated spectral problem on $[0, b]$ for some $b>0$ with the Dirichlet boundary condition at $b$ and purely discrete spectrum, and then passing to the limit $b \rightarrow \infty$ (this approach is detailed in Linetsky 2004a).

\subsection{Pricing $L^{2}$ Payoffs}

We now apply the spectral representation to the valuation problem (3.5). Let $\mathcal{H}:=$ $L^{2}((0, \infty), \mathfrak{m})$ be the Hilbert space of functions square-integrable with the speed density $\mathfrak{m}$ in equation (3.8) and endowed with the inner product

$$
(f, g)_{\mathfrak{m}}=\int_{0}^{\infty} f(x) g(x) \mathfrak{m}(x) d x .
$$

For any $\psi$ such that $\chi_{\psi} \in \mathcal{H}\left(\chi_{\psi}\right.$ is defined in (3.6)), the valuation (3.5) has the spectral representation

$$
\begin{aligned}
e^{q T} S^{-1} V_{\psi}(S, T)= & \frac{1}{2 \pi^{2}} \int_{0}^{\infty} e^{-\frac{\left(\nu^{2}+\rho^{2}\right) \tau}{2}} C_{\psi}(\rho) x^{\frac{1-v}{2}} e^{\frac{1}{4 x}} \\
& \times W_{\frac{1-v}{2}, \frac{i \rho}{2}}\left(\frac{1}{2 x}\right)\left|\Gamma\left(\frac{v+i \rho}{2}\right)\right|^{2} \sinh (\pi \rho) \rho d \rho \\
& +\mathbf{1}_{\{v<0\}} c_{\psi}(0)+\mathbf{1}_{\{v<-2\}} \sum_{n=1}^{[|\nu| / 2]} c_{\psi}(n) e^{-2 n(|v|-n) \tau} \\
& \times \frac{2(|\nu|-2 n) n !}{\Gamma(1+|v|-n)}(2 x)^{n} L_{n}^{(|\nu|-2 n)}\left(\frac{1}{2 x}\right),
\end{aligned}
$$

where the expansion coefficients are given by

$$
C_{\psi}(\rho)=\int_{0}^{\infty} y^{\frac{\nu-1}{2}} e^{-\frac{1}{4 y}} W_{\frac{1-v}{2}, \frac{i \rho}{2}}\left(\frac{1}{2 y}\right) \chi_{\psi}(y) d y,
$$

$$
c_{\psi}(0)=\int_{0}^{\infty} \chi_{\psi}(y) \pi(y) d y, \quad c_{\psi}(n)=\int_{0}^{\infty}(2 y)^{n-|\nu|-1} e^{-\frac{1}{2 y}} L_{n}^{(|\nu|-2 n)}\left(\frac{1}{2 y}\right) \chi_{\psi}(y) d y .
$$


For details of the spectral expansion approach to derivatives pricing, see Linetsky (2004b) and references therein.

\subsection{Pricing Bonds}

First consider the case $v<2 / p$ (from equation (3.3) this condition is equivalent to $\left.r-q-\sigma^{2} / 2<0\right)$. Bond payoff $\psi_{\text {bond }}(x)=1$ is such that $\chi_{\psi} \in \mathcal{H}$, and the spectral representation (3.13) is applicable. Fortunately, in this case the integrals in equations (3.14) and (3.15) can be calculated in closed form.

The case $v \geq 2 / p$ (equivalently, $r-q-\sigma^{2} / 2 \geq 0$ ) is more involved. For the bond payoff $\psi_{\text {bond }}(x)=1, \chi_{\psi}(x)=(x / \beta)^{-1 / p} \notin \mathcal{H}$ and, hence, the spectral representation (3.13) cannot be applied. The alternative is to first compute the Laplace transform

$$
\Phi_{s}^{(v)}(x):=\int_{0}^{\infty} e^{-s \tau} E_{x}^{(v)}\left[\left(X_{\tau}^{(v)} / \beta\right)^{-1 / p}\right] d \tau=\int_{0}^{\infty}(y / \beta)^{-1 / p} G_{s}^{(v)}(x, y) d y
$$

with the kernel (3.10) and then do the Laplace inversion, choosing the contour of integration in the Bromwich complex Laplace inversion formula to the right of any singularities of $\Phi_{s}^{(v)}(x)$ in the complex s-plane. In this case the function $\Phi_{s}^{(v)}(x)$ has an additional pole in addition to the singularities inherited from the Green's function $G_{s}^{(v)}(x, y)$, resulting in an additional positive term in the pricing formula for $v>2 / p$ (this phenomenon for non- $L^{2}$ payoffs has been first discussed in Lewis 1998). The interpretation of this additional term as the discounted probability of the firm asymptotically escaping to large stock price values and ultimately avoiding bankruptcy is provided in Section 4.

Proposition 3.4. The pricing function (2.8) for the zero-coupon bond with unit face value and no recovery is given by

$$
\begin{aligned}
B(S, T)= & \mathbf{1}_{\{v>2 / p\}} e^{-r T} \frac{\Gamma(v-1 / p)}{\Gamma(v-2 / p)} U\left(\frac{1}{p}, \frac{2}{p}-v+1, \frac{1}{2 x}\right)+\mathbf{1}_{\{v<0\}} e^{-q T} \frac{\Gamma(1 / p-v)}{\Gamma(-v)}(2 x)^{\frac{1}{p}} \\
& +\mathbf{1}_{\{v<-2\}} e^{-q T} \sum_{n=1}^{[|v| / 2]} e^{-2 n(|v|-n) \tau} \frac{(|v|-2 n) \Gamma(-1 / p) \Gamma(1 / p+|v|-n)}{\Gamma(1+|v|-n) \Gamma(1-1 / p-n)} \\
& \times(2 x)^{\frac{1}{p}+n} L_{n}^{(|v|-2 n)}\left(\frac{1}{2 x}\right)+\frac{e^{-q T}}{4 \pi^{2} \Gamma(1 / p)} \int_{0}^{\infty} e^{-\frac{\left(v^{2}+\rho^{2}\right) \tau}{2}}(2 x)^{\frac{1}{p}+\frac{1-v}{2}} e^{\frac{1}{4 x}} \\
& \times W_{\frac{1-v}{2}, \frac{i \rho}{2}}\left(\frac{1}{2 x}\right)\left|\Gamma\left(\frac{v+i \rho}{2}\right) \Gamma\left(\frac{1}{p}-\frac{v+i \rho}{2}\right)\right|^{2} \sinh (\pi \rho) \rho d \rho,
\end{aligned}
$$

where $U(a, b, z)$ is the second confluent hypergeometric function (see Appendix $C$ ).

Proof. See Appendix D.

\subsection{Pricing Options}

The put payoff $\psi(x)=(K-x)^{+}$is such that $\chi_{\psi} \in \mathcal{H}$ for all $v \in \mathbb{R}$ and, hence, the valuation of the put payoff given no bankruptcy (2.6) follows from the spectral expansion (3.13). Fortunately, the integrals in the expressions for the expansion coefficients (3.14) and (3.15) can be computed in closed form. The recovery part (bankruptcy claim) value (2.7) follows from the bond valuation in Proposition 3.4. 
Proposition 3.5. Define $k=\beta K^{p}$. The put pricing function (2.4c) is given by

$$
\begin{aligned}
P_{K}(S, T)= & e^{-r T} K-\mathbf{1}_{\{v>2 / p\}} e^{-r T} K \frac{\Gamma(v-1 / p)}{\Gamma(v-2 / p)} U\left(\frac{1}{p}, \frac{2}{p}-v+1, \frac{1}{2 x}\right) \\
& -\mathbf{1}_{\{v<0\}} \frac{e^{-q T} S}{\Gamma(|v|)}\left[\Gamma\left(|v|, \frac{1}{2 k}\right)+(2 k)^{\frac{1}{p}} \gamma\left(\frac{1}{p}+|v|, \frac{1}{2 k}\right)\right] \\
& -\mathbf{1}_{\{v<-2\}} e^{-q T} S \sum_{n=1}^{[|v| / 2]} p_{K}(n) e^{-2 n(|v|-n) \tau} \frac{2(|v|-2 n) n !}{\Gamma(1+|v|-n)}(2 x)^{n} L_{n}^{(|v|-2 n)}\left(\frac{1}{2 x}\right) \\
& -e^{-q T} S \frac{1}{2 \pi^{2}} \int_{0}^{\infty} P_{K}(\rho) e^{-\frac{\left(v^{2}+\rho^{2}\right) \tau}{2}} x^{\frac{1-v}{2}} e^{\frac{1}{4 x}} \\
& \times W_{\frac{1-v}{2}, \frac{i \rho}{2}}\left(\frac{1}{2 x}\right)\left|\Gamma\left(\frac{v+i \rho}{2}\right)\right|^{2} \sinh (\pi \rho) \rho d \rho,
\end{aligned}
$$

where

$$
\begin{aligned}
P_{K}(\rho)= & k^{\frac{1+v}{2}} e^{-\frac{1}{4 k}} W_{-\frac{1+v}{2}}, \frac{i \rho}{2}\left(\frac{1}{2 k}\right)+2 \mathfrak{R}\left\{\frac{(2 k)^{\frac{v-i \rho}{2}}(2 / p-v-i \rho) \Gamma(-i \rho)}{\Gamma(v / 2-i \rho / 2)\left(\rho^{2}+(v-2 / p)^{2}\right)}\right. \\
& \left.\times{ }_{2} F_{2}\left[\frac{1}{p}-\frac{v}{2}+\frac{i \rho}{2}, 1-\frac{v}{2}+\frac{i \rho}{2} ; 1+i \rho, \frac{1}{p}-\frac{v}{2}+\frac{i \rho}{2}+1 ;-\frac{1}{2 k}\right]\right\}, \\
p_{K}(n)= & -\frac{1}{2 n}(2 k)^{n-|v|} e^{-\frac{1}{2 k}} L_{n-1}^{(|v|-2 n)}\left(\frac{1}{2 k}\right)+\frac{\Gamma(|v|-n+1)(2 k)^{n-|v|}}{2(|v|-n+1 / p) n ! \Gamma(|v|-2 n+1)} \\
& \times{ }_{2} F_{2}\left[|v|-n+1,|v|-n+\frac{1}{p} ;|v|-2 n+1,|v|-n+1+\frac{1}{p} ;-\frac{1}{2 k}\right],
\end{aligned}
$$

and ${ }_{2} F_{2}\left[a_{1}, a_{2} ; b_{1}, b_{2} ; z\right]$ is the hypergeometric function given in (C.13), $\gamma(a, x)=$ $\int_{0}^{x} z^{a-1} e^{-z} d z$ is the incomplete Gamma function, $\Gamma(a, x)=\int_{x}^{\infty} z^{a-1} e^{-z} d z$ is the complementary incomplete Gamma function, and $\Re(z) \equiv(z+\bar{z}) / 2$ denotes the real part of a complex number $z$.

\section{Proof. See Appendix D.}

The call payoff $\psi_{\text {call }}(x)=(x-K)^{+}$is such that $\chi_{\psi} \in \mathcal{H}$ for $v<0$ but $\chi_{\psi} \notin \mathcal{H}$ for $v \geq$ 0 . Nevertheless, we can use the put-call parity (2.5) to recover the call pricing function $C_{K}(S, T)$ for all $v \in \mathbb{R}$ from the put pricing formula in Proposition 3.5.

\section{ECONOMIC ANALYSIS OF THE MODEL: CREDIT SPREADS AND IMPLIED VOLATILITY SKEWS}

Assuming the unit face value $T$-maturity zero-coupon bond recovers nothing in the event of bankruptcy $(R=0)$, the $T$-maturity credit spread is defined as usual

$$
\mathcal{S}(S, T)=-\frac{1}{T} \ln B(S, T)-r .
$$

For small $T$, asymptotically we have

$$
\mathcal{S}(S, T) \sim h(S) \quad \text { as } \quad T \rightarrow 0 .
$$


To investigate the long maturity asymptotics of the term structure of credit spreads, define the asymptotic credit spread as

$$
\mathcal{S}_{\infty}:=\lim _{T \rightarrow \infty} \mathcal{S}(S, T)
$$

There are three distinct cases, depending on the relationship among the risk-free rate, dividend yield, and volatility.

(i) When $|r-q| \leq \sigma^{2} / 2(0 \leq v \leq 2 / p)$, the spectrum is purely continuous, the bond pricing function in Proposition 3.4 contains only the integral term, and we have (recall that $\tau=p^{2} \sigma^{2} T / 4$ )

$$
\mathcal{S}_{\infty}=q-r+\frac{v^{2} p^{2} \sigma^{2}}{8}=\frac{1}{2 \sigma^{2}}\left(r-q-\frac{\sigma^{2}}{2}\right)^{2} .
$$

For large $T$, the term structure of credit spreads flattens out toward the asymptotic spread $\mathcal{S}_{\infty}=\left(r-q-\sigma^{2} / 2\right)^{2} /\left(2 \sigma^{2}\right) \in\left[0, \sigma^{2} / 2\right]$. Note that it depends only on $r, q$ and $\sigma$, and not on the hazard rate parameters $\alpha$ and $p$.

(ii) When the dividend yield is sufficiently large so that $q>r+\sigma^{2} / 2(v<0)$, there is some nonempty discrete spectrum containing at least the zero principal eigenvalue that contributes the leading term in the spectral expansion of the bond pricing function, and

$$
\mathcal{S}_{\infty}=q-r .
$$

For large $T$, the term structure of credit spreads flattens out toward the asymptotic spread $\mathcal{S}_{\infty}=q-r>\sigma^{2} / 2$. In this case it depends only on $r$ and $q$.

(iii) When the risk-free rate is sufficiently large so that $r>q+\sigma^{2} / 2(v>2 / p)$, the bond pricing function contains an additional term

$$
e^{-r T} \frac{\Gamma(v-1 / p)}{\Gamma(v-2 / p)} U\left(\frac{1}{p}, \frac{2}{p}-v+1, \frac{1}{2 x}\right)
$$

and

$$
\mathcal{S}_{\infty}=0
$$

In this case, asymptotically the term structure of credit spreads declines to zero for long maturities. To understand this behavior, consider the process (2.1). For large values of the stock price, the process behaves asymptotically as GBM. The natural boundary at infinity is attracting when $r-q-\sigma^{2} / 2>0$. For $r-q-\sigma^{2} / 2>0$, there is thus a positive probability that the stock price process asymptotically escapes to large values and the firm never goes bankrupt:

$$
\mathbb{P}\left(\lim _{t \rightarrow \infty} S_{t}^{\Delta}=\infty \mid S_{0}=S\right)>0 .
$$

We can calculate this (risk-neutral) probability directly.

Proposition 4.1. For $v>2 / p$ (equivalently, $r-q-\sigma^{2} / 2>0$ ) and $S>0$,

$$
\mathbb{P}\left(\lim _{t \rightarrow \infty} S_{t}^{\Delta}=\infty \mid S_{0}=S\right)=\frac{\Gamma(v-1 / p)}{\Gamma(v-2 / p)} U\left(\frac{1}{p}, \frac{2}{p}-v+1, \frac{1}{2 x}\right) .
$$


Term Structure of Credit Spreads

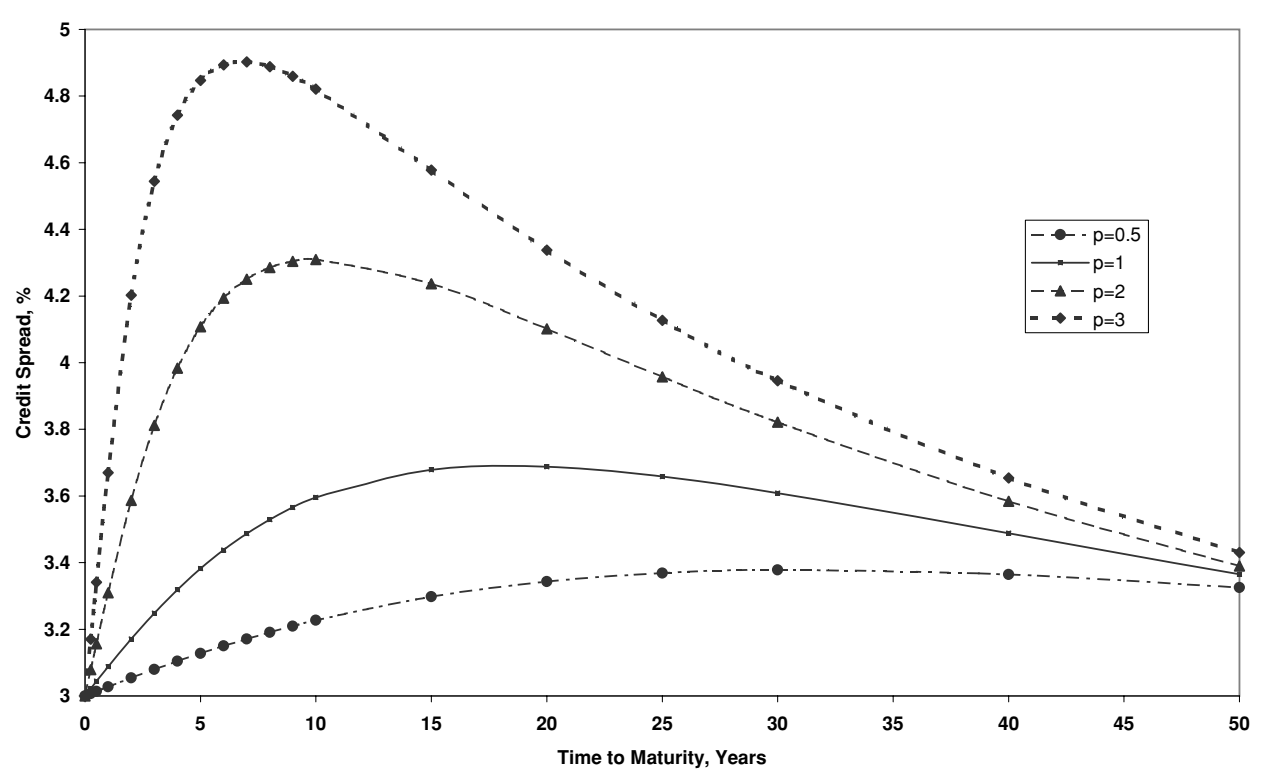

FIgURE 4.1. Term structure of credit spreads. Parameter values: $S=S^{*}=50, \sigma=$ $0.3, r=q=0.03, h^{*}=0.03, p=0.5,1,2,3$.

\section{Proof. See Appendix D.}

For the bond price, we have the large- $T$ asymptotics

$$
B(S, T) \sim e^{-r T} \mathbb{P}\left(\lim _{t \rightarrow \infty} S_{t}^{\Delta}=\infty \mid S_{0}=S\right) \quad \text { as } \quad T \rightarrow \infty .
$$

Therefore, the additional term in the bond pricing function $B(S, T)$ for $v>2 / p$ is the discounted (risk-neutral) probability of the firm asymptotically escaping to large stock price values and ultimately avoiding bankruptcy.

We now investigate the possible shapes of the term structure of credit spreads. It is convenient to parameterize the hazard rate as follows:

$$
h(S)=h^{*}\left(\frac{S^{*}}{S}\right)^{p},
$$

where $S^{*}>0$ is some reference stock price level and $h^{*}>0$ is the hazard rate at that reference level, $h\left(S^{*}\right)=h^{*}$, so that $h^{*}$ serves as the scale parameter. To calibrate the model in applications, one typically selects $S^{*}=S_{0}$, the initial stock price at the time of calibration.

Figures 4.1-4.3 illustrate the shapes of the term structure of credit spreads for the three cases considered above. ${ }^{4}$ Volatility is fixed at $30 \%(\sigma=0.3)$. Figure 4.1 gives an example of the first case. The risk-free rate and the dividend yield are both equal to $3 \%(r=q=0.03)$.

\footnotetext{
${ }^{4}$ For all numerical computations in this paper we used Mathematica software package running on a PC. All required special functions are available in Mathematica as standard built-in functions. The single integral with respect to the spectral parameter $\rho$ in the valuation formulas was computed using the built-in numerical integration routine in Mathematica.
} 
Term Structure of Credit Spreads

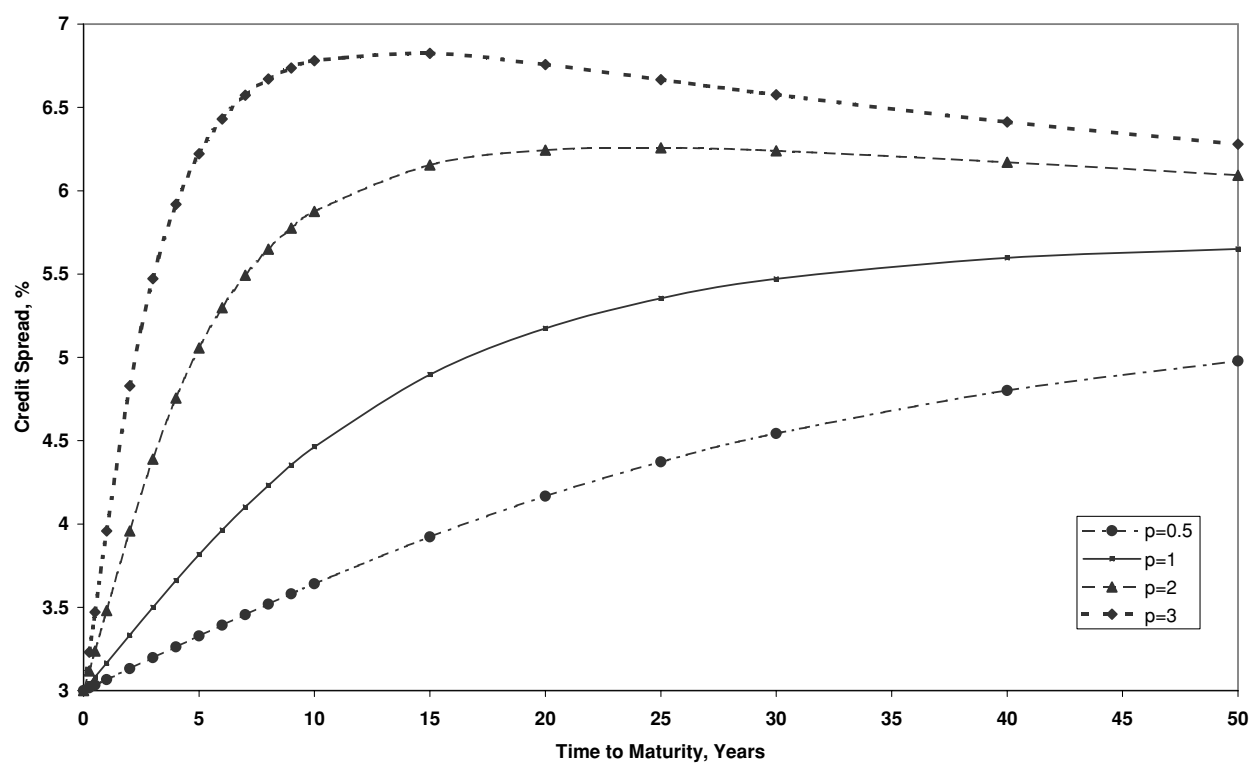

FiguRE 4.2. Term structure of credit spreads. Parameter values: $S=S^{*}=50, \sigma=$ $0.3, r=0.02, q=0.07, h^{*}=0.03, p=0.5,1,2,3$.

Term Structure of Credit Spreads

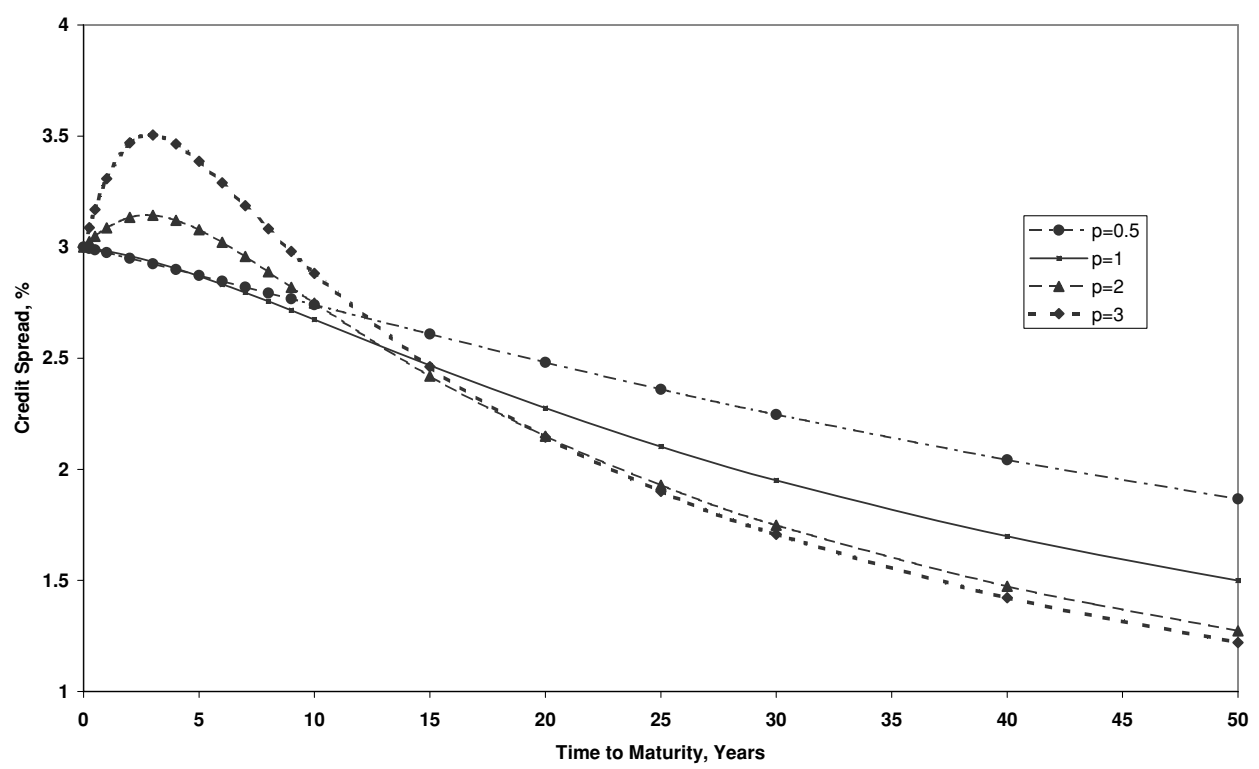

FiguRE 4.3. Term structure of credit spreads. Parameter values: $S=S^{*}=50, \sigma=$ $0.3, r=0.07, q=0, h^{*}=0.03, p=0.5,1,2,3$. 
In this case $|r-q| \leq \sigma^{2} / 2$ and the asymptotic spread is $\mathcal{S}_{\infty}=0.01125$ (112.5 basis points). Figure 4.1 plots four curves corresponding to the four choices of the hazard rate parameter $p=0.5,1,2,3$ with $h^{*}=0.03$ and $S^{*}=50$. For these parameter values, the term structure has a humped shape, first upward slopping and then slowly downward slopping toward the asymptotic yield.

Figure 4.2 gives an example of the second case with $r=0.02$ and $q=0.07$. In this case $q>$ $r+\sigma^{2} / 2$ and the asymptotic spread is $\mathcal{S}_{\infty}=0.05$ (500 basis points). Figure 4.2 plots four curves corresponding to the four choices of the hazard rate parameter $p=0.5,1,2,3$ with $h^{*}=0.03$ and $S^{*}=50$. The term structures are upward slopping, have a hump, and then decline toward the asymptotic spread of $5 \%$, which is much larger than that in Figure 4.1 since the dividend yield is $q>r+\sigma^{2} / 2$.

Figure 4.3 gives an example of the third case with $r=0.07$ and $q=0$. In this case $r>$ $q+\sigma^{2} / 2$ and the asymptotic spread is $\mathcal{S}_{\infty}=0$. Figure 4.3 plots four curves corresponding to the four choices of the hazard rate parameter $p=0.5,1,2,3$ with $h^{*}=0.03$ and $S^{*}=50$. The terms structures are downward slopping toward zero. The term structures with $p=2$ and 3 have an initial hump. To further illustrate this case, Figure 4.4 plots the (risk-neutral) probability of the firm escaping to the high stock price values and ultimately avoiding bankruptcy as a function of the initial stock price $u(S)=\mathbb{P}\left(\lim _{t \rightarrow \infty} S_{t}^{\Delta}=\infty \mid S_{0}=S\right)$. Note that this probability increases with $p$. Since $r$ is large and $q=0$, the stock price tends to appreciate. For larger values of $p$, the hazard rate falls off more quickly as the stock price increases, thus increasing the probability of avoiding bankruptcy.

Figure 4.5 plots the option implied volatility against the strike price of the option in the model with $S^{*}=50, \sigma=0.3, r=q=0.03, h^{*}=0.03$, and $p=2$ for expirations of 3 months, 6 months, 1 year, and 5 years (these implied volatility curves correspond to the term structure of credit spreads in Figure 4.1 with $p=2$ ). The implied volatilities are obtained by first computing the put pricing formula of Proposition 3.5 for a given strike and expiration and then implying the Black-Scholes implied volatility. The current stock price is $S=50$. For higher strikes, the implied volatility asymptotically decreases toward $\sigma=0.3$, as the hazard rate vanishes and the process becomes asymptotically GBM as the stock price increases. For lower strike prices, we observe the characteristic implied

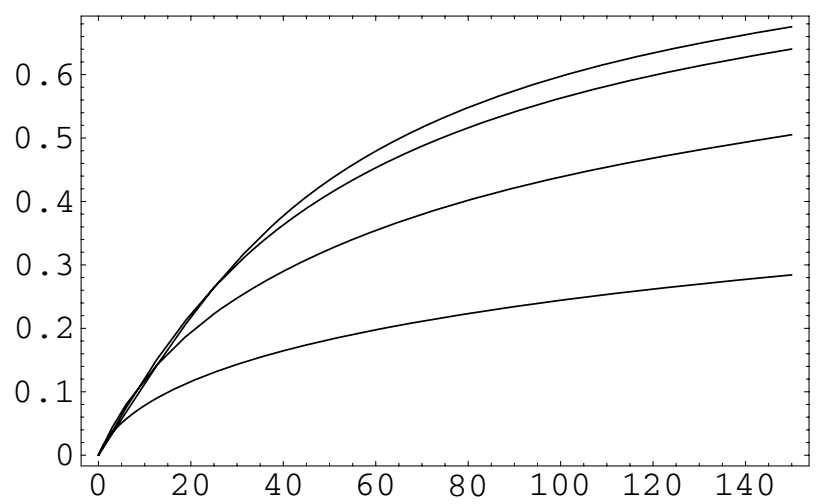

FIgURE 4.4. Probability of avoiding bankruptcy as a function of the initial stock price, $u(S)=\mathbb{P}\left(\lim _{t \rightarrow \infty} S_{t}^{\Delta}=\infty \mid S_{0}=S\right)$. Parameter values: $S^{*}=50, \sigma=0.3, r=$ $0.07, q=0, h^{*}=0.03, p=0.5,1,2,3$ (the lower curve corresponds to $p=0.5$, the upper curve corresponds to $p=3$ ). 


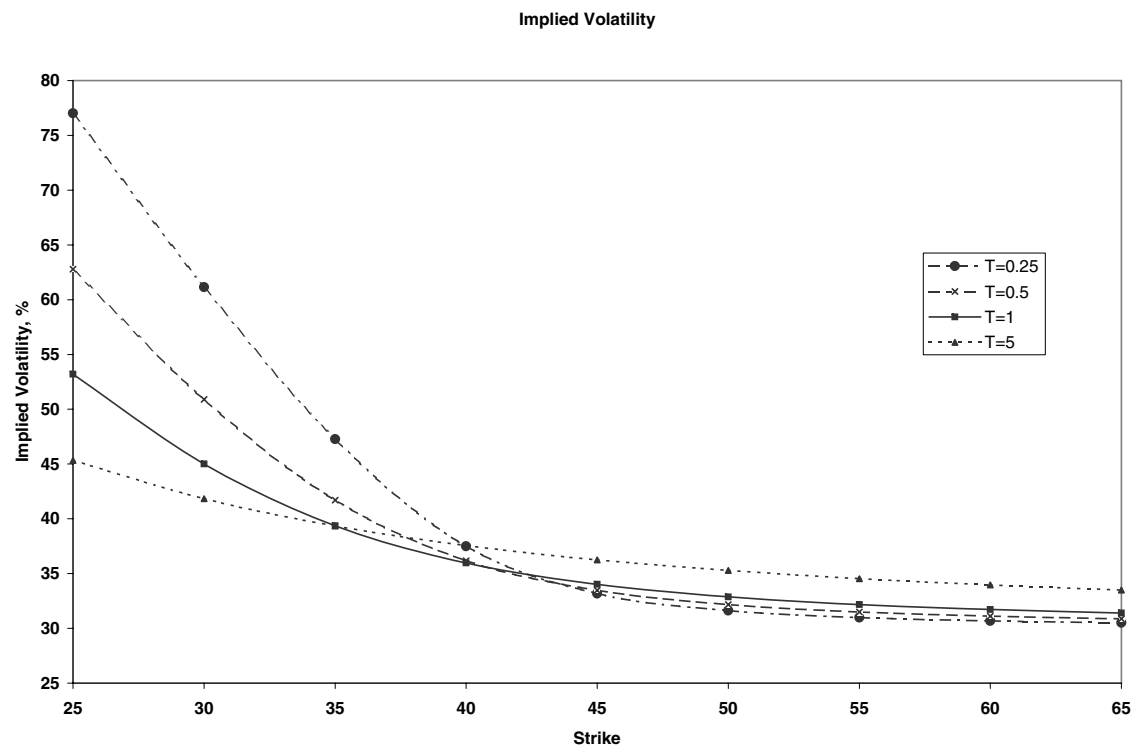

FIgURE 4.5. Implied volatilities for times to expiration $T=0.25,0.5,1,5$. Parameter values: $S=S^{*}=50, \sigma=0.3, r=q=0.03, h^{*}=0.03, p=2$.

volatility skew with implied volatilities increasing for lower strikes, as the hazard rate increases as the stock price declines. Moreover, shorter expirations exhibit steeper skews and the skews gradually flatten out for longer maturities. For larger values of $p$, the skew is steeper for out-of-the-money puts, since the hazard rate increases more rapidly as the stock price declines. On the other hand, for larger $p$, implied volatilities approach the

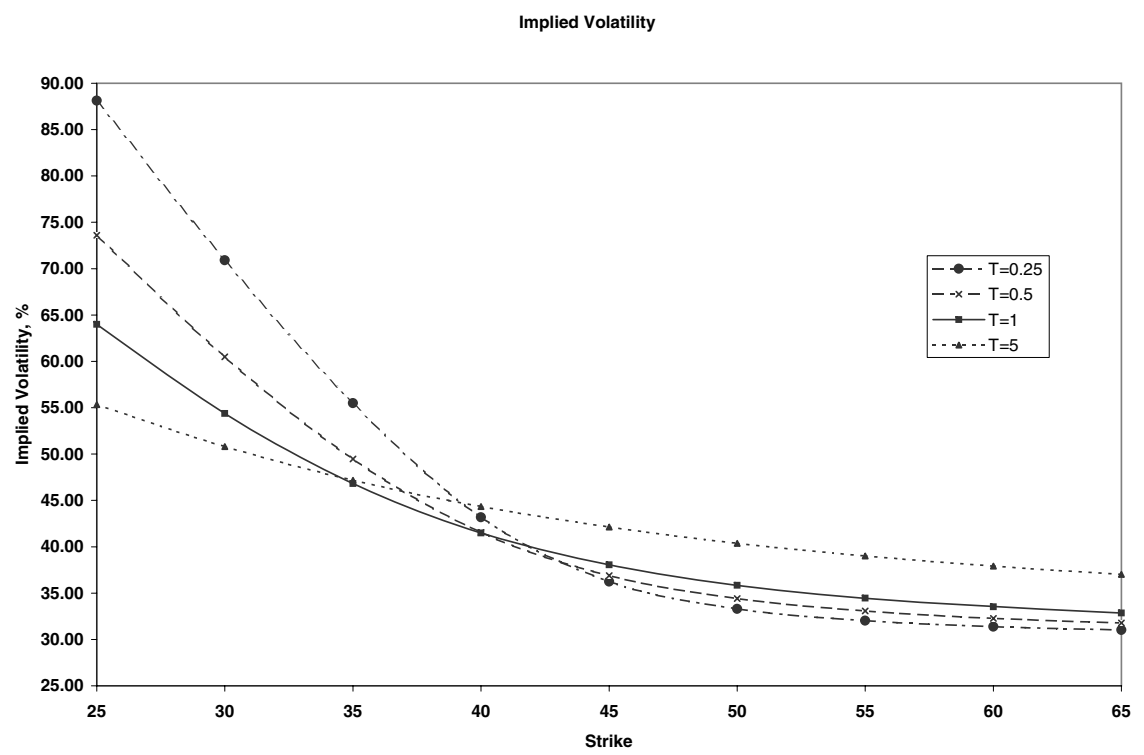

FIGURE 4.6. Implied volatilities for times to expiration $T=0.25,0.5,1,5$. Parameter values: $S=S^{*}=50, \sigma=0.3, r=q=0.03, h^{*}=0.06, p=2$. 
bankruptcy-free volatility $\sigma$ more rapidly for out-of-the-money calls, as the hazard rate falls off more rapidly as the stock price increases. For smaller $p$, the skew is flatter, but declines toward the bankruptcy-free $\sigma$ slower.

Figure 4.6 plots implied volatility skews for the case with $h^{*}=0.06$. We observe that as the hazard rate parameter $h^{*}$ increases, the skews become steeper. We thus have a clear link between the hazard rate of bankruptcy and resulting credit spreads and option implied volatility skews. Increasing probability of bankruptcy of the underlying firm increases both credit spreads on corporate bonds and implied volatility skews in stock options.

\section{CONCLUSION}

In this paper we have solved in closed form a parsimonious extension of the BlackScholes-Merton model with bankruptcy where the hazard rate of bankruptcy is a negative power of the stock price. By combining a scale change and a measure change, we reduced the model dynamics to a linear stochastic differential equation, whose solution is a diffusion process that has played a central role in the pricing of Asian options. The solution is in the form of a spectral expansion associated with the diffusion infinitesimal generator. Pricing formulas for both corporate bonds and stock options are obtained in closed form. Term credit spreads on corporate bonds and implied volatility skews of stock options are closely linked in this model, with parameters of the hazard rate specification controlling both the shape of the term structure of credit spreads and the slope of the implied volatility skew. The results of our analysis provide further insights into the linkage between corporate credit spreads and volatility skews in stock options. Our analytical formulas are easy to implement and, it is hoped, will prove useful to researchers and practitioners in corporate debt and equity derivatives markets.

To conclude, we note that credit risk is not the only cause of implied volatility skews in equity options. Stochastic volatility $\sigma$ that is negatively correlated with the stock price process will further contribute to the steepening of the skew. In this paper we kept $\sigma$ constant to focus on the credit risk aspect of the problem. Carr and Linetsky (2005) study a jump-to-default extension of the constant elasticity of variance (CEV) model, where $\sigma$ is a function the underlying stock price.

\section{APPENDIX A: MATHEMATICAL ORIGINS OF THE DENSITY $p^{(v)}$ : BROWNIAN EXPONENTIAL FUNCTIONALS, SCHRÖDINGER OPERATOR WITH MORSE POTENTIAL, AND MAASS LAPLACIAN}

In this appendix we discuss the mathematical origins of the density $p^{(v)}$ in Proposition 3.3 and provide relevant references. For $v<0$, this density was first obtained by Wong (1964, p. 271, equation 38) (see also Comtet, Monthus, and Yor [1998] and Linetsky [2004a]). This density is closely related to several classical mathematical objects.

For $v \in \mathbb{R}$, consider the process $X^{(v)}$ solving the SDE (3.4) and starting at $x>0$. Define a new process $\left\{Z_{t}^{(v)}:=\frac{1}{2} \ln X_{t}^{(v)}, t \geq 0\right\}$,

$$
d Z_{t}^{(v)}=\left(v+\frac{1}{2} e^{-2 Z_{t}^{(v)}}\right) d t+d B_{t}, \quad Z_{0}^{(v)}=z=\frac{1}{2} \ln x .
$$

Let $P_{z}^{(v)}$ be the law of the process $Z^{(v)}$ starting at $z \in \mathbb{R}$ and let $P_{z}^{B}$ be the law of standard Brownian motion starting at $z \in \mathbb{R}$. 
Proposition A.1. We have the following absolute continuity relationship $\left.\frac{d P_{z}^{(v)}}{d P_{z}^{B}}\right|_{\mathcal{F}_{t}}=\exp \left\{-\frac{v^{2}}{2} t+v\left(B_{t}-z\right)-\frac{1}{4}\left(e^{-2 B_{t}}-e^{-2 z}\right)-\int_{0}^{t}\left(\frac{v-1}{2} e^{-2 B_{u}}+\frac{1}{8} e^{-4 B_{u}}\right) d u\right\}$.

Proof. This result follows from Girsanov's theorem.

Let $q^{(v)}(t ; x, y)$ be the density defined by

$$
q^{(v)}(t ; x, y):=\frac{\partial}{\partial y} E_{x}^{B}\left[e^{-\int_{0}^{t}\left(\frac{v-1}{2} e^{-2 B_{u}}+\frac{1}{8} e^{-4 B_{u}}\right) d u} \mathbf{1}_{\left\{B_{t} \leq y\right\}}\right],
$$

where $B$ is a standard Brownian motion starting at $x$. From Proposition A.1 we have

$$
p^{(v)}(t ; x, y)=e^{-\frac{v^{2}}{2} t} e^{\frac{1}{4 x}-\frac{1}{4 y}}\left(\frac{y}{x}\right)^{\frac{v}{2}} q^{(v)}\left(t ; \frac{1}{2} \ln x, \frac{1}{2} \ln y\right) .
$$

By the Feynman-Kac theorem, the density $q^{(v)}(t ; x, y)$ is the heat kernel of the secondorder differential operator

$$
\mathcal{H}^{(v)}=-\frac{1}{2} \frac{d^{2}}{d x^{2}}+\frac{v-1}{2} e^{-2 x}+\frac{1}{8} e^{-4 x},
$$

a self-adjoint operator in $L^{2}(\mathbb{R})$. The heat kernel satisfies the heat equation with the operator $\mathcal{H}^{(v)}$

$$
\mathcal{H}^{(v)} q=-\frac{\partial q}{\partial t}
$$

with the initial condition $q^{(v)}(0 ; x, y)=\delta(x-y)$, where $\delta(\cdot)$ is Dirac's delta. The operator $\mathcal{H}^{(v)}$ is the well-known Schrödinger operator with Morse potential. The spectral expansion of the density $p^{(v)}$ in Proposition 3.3 thus follows from the spectral expansion of the Schrödinger operator with Morse potential. The Schrödinger operator

$$
-\frac{d^{2}}{d x^{2}}+V(x)
$$

with potential of the form

$$
V(x)=a e^{-\beta x}+b e^{-2 \beta x}
$$

first appeared in quantum mechanics in the classical paper Morse (1929) on the spectra of diatomic molecules. It is closely related to another classical differential operator, the Maass Laplacian or Schrödinger operator on the Poincaré upper half-plane in a magnetic field.

Let $\mathbb{H}^{2}$ be the upper half-plane with rectangular coordinates $(x, y), x \in \mathbb{R}, y>0$, and with the Poincaré metric (hyperbolic plane). Consider the Schrödinger operator with a uniform magnetic field $B, B \in \mathbb{R}$, on $\mathbb{H}^{2}$

$$
\mathcal{H}_{B}=-\frac{1}{2} y^{2}\left(\frac{\partial^{2}}{\partial x^{2}}+\frac{\partial^{2}}{\partial y^{2}}\right)+i B y \frac{\partial}{\partial x}+\frac{B^{2}}{2} .
$$

This is a $-1 / 2$ of the standard Laplace-Beltrami operator on $\mathbb{H}^{2}$ plus magnetic field terms. Introduce a new variable $\eta=-\frac{1}{2} \ln y$. On functions of the form $u(x, \eta)=\exp (-i p x-$ $\left.\frac{1}{2} \eta\right) v(\eta)$ the operator $\mathcal{H}_{B}$ reduces to the Schrödinger operator with Morse potential. 
Harmonic analysis on the hyperbolic plane can be applied to obtain its spectral representation.

Thus, the density of linear diffusion (3.4), the density of Brownian motion killed at a linear combination of two Brownian exponential functionals (A.1), the heat kernel of the Schrödinger operator with Morse potential, and the heat kernel of the Maass Laplacian on the hyperbolic plane are closely related. These connections have been explored in Alili and Gruet (1997), Alili, Matsumoto, and Shiraishi (2001), Comtet (1987), Comtet and Monthus (1996), Comtet, Monthus, and Yor (1998), Grosche (1988), and Ikeda and Matsumoto (1999) in several different contexts.

\section{APPENDIX B: CONNECTION WITH ASIAN OPTIONS}

The process $X^{(v)}$ and its density $p^{(v)}$ are closely related to the problem of pricing arithmetic Asian options. Assume that, under the EMM, the underlying asset price follows a GBM process $\left\{S_{t}=S_{0} \exp \left(\sigma B_{t}+\left(r-q-\sigma^{2} / 2\right) t\right), t \geq 0\right\}$. For $t>0$, let $\mathcal{A}_{t}$ be the continuous arithmetic average price, $\mathcal{A}_{t}=t^{-1} \int_{0}^{t} S_{u} d u$. An Asian call (put) option with strike $K>$ 0 and expiration $t>0$ delivers the payoff $\left(\mathcal{A}_{T}-K\right)^{+}\left(\left(K-\mathcal{A}_{T}\right)^{+}\right)$at $T$. After standardizing the problem (see Geman and Yor 1993), it reduces to computing expectations of the form $E\left[\left(A_{\tau}^{(v)}-k\right)^{+}\right]\left(E\left[\left(k-A_{\tau}^{(v)}\right)^{+}\right]\right)$, where $\tau=\sigma^{2} T / 4, v=2\left(r-q-\sigma^{2} / 2\right) / \sigma^{2}, k=$ $\tau K / S_{0}$, and $A_{\tau}^{(v)}$ is a Brownian exponential functional (see Yor 2001)

$$
A_{\tau}^{(v)}=\int_{0}^{\tau} e^{2\left(B_{u}+v u\right)} d u
$$

Dufresne's identity in law (Dufresne 1989, 1990; see also Donati-Martin, Ghomrasni, and Yor 2001) states that, for each fixed $t>0$,

$$
A_{t}^{(v)} \stackrel{\text { (law) }}{=} X_{t}^{(v)}
$$

where $X_{t}^{(v)}$ is the diffusion process (3.4) starting at the origin. To see this, recall equation (3.3) (in this case $x=0$ ). By invariance to time reversal of Brownian motion, for each fixed $t>0$

$$
X_{t}^{(v)}=\int_{0}^{t} e^{2\left(B_{t}-B_{u}\right)+2 v(t-u)} d u \stackrel{(\text { law })}{=} \int_{0}^{t} e^{2\left(B_{s}+v s\right)} d s=A_{t}^{(v)} .
$$

Dufresne's identity in law was applied to the valuation of Asian options by DonatiMartin, Ghomrasni, and Yor (2001). To compute the Asian option price, these authors observe that this computation is equivalent to the computation of the price of an option written on the process $X^{(v)}$ starting at the origin. They compute the resolvent kernel of $X^{(v)}$ and, on integration with the payoff, obtain the Laplace transform of the option price with respect to time to expiration. This gives an alternative derivation of the celebrated Geman and Yor $(1992,1993)$ Laplace transform result (which was originally obtained via Lamperti's identity and the theory of Bessel processes). To recover the Asian option price for fixed time to expiration, one needs to invert the Laplace transform. The Laplace inversion for Asian options is accomplished in Linetsky (2004a) by means of the spectral expansion approach.

\section{APPENDIX C: CONFLUENT HYPERGEOMETRIC FUNCTIONS}

This appendix collects some facts about the confluent hypergeometric functions. The reader is referred to Slater (1960), Buchholz (1969), Abramowitz and Stegun (1972), and 
Prudnikov, Brychkov, and Marichev (1990) for further details. All the special functions in this Appendix are available as built-in functions in Mathematica and Maple software packages. To compute these functions efficiently, these packages use a variety of integral and asymptotic representations given in the above references, in addition to the defining hypergeometric series presented here.

The Kummer confluent hypergeometric function is defined by the hypergeometric series

$$
{ }_{1} F_{1}[a ; b ; z]=\sum_{n=0}^{\infty} \frac{(a)_{n}}{(b)_{n}} \frac{z^{n}}{n !},
$$

where $(a)_{0}=1,(a)_{n}=a(a+1) \cdots(a+n-1)$ are the Pochhammer symbols, $(a)_{n}=$ $\Gamma(a+n) / \Gamma(a)$, where $\Gamma(z)$ is the Gamma function. The regularized Kummer function ${ }_{1} F_{1}[a ; b ; z] / \Gamma(b)$ is an analytic function of $a, b$, and $z$, and is defined for all values of $a, b$, and $z$ real or complex. The second confluent hypergeometric function (Tricomi function) is defined by

$$
U(a, b, z)=\frac{\pi}{\sin (\pi b)}\left\{\frac{{ }_{1} F_{1}[a ; b ; z]}{\Gamma(1+a-b) \Gamma(b)}-\frac{z^{1-b}{ }_{1} F_{1}[1+a-b ; 2-b ; z]}{\Gamma(a) \Gamma(2-b)}\right\} .
$$

It is analytic for all values of $a, b$, and $z$ real or complex even when $b$ is zero or a negative integer, for in these cases it can be defined in the limit $b \rightarrow \pm n$ or 0 . It has the following symmetry property

$$
U(a, b, z)=z^{1-b} U(1+a-b, 2-b, z) .
$$

The confluent hypergeometric functions are solutions of the confluent hypergeometric equation

$$
z \frac{d^{2} u}{d z^{2}}+(b-z) \frac{d u}{d z}-a u=0
$$

The first Whittaker function is defined by

$$
M_{\kappa, \mu}(z)=z^{\mu+1 / 2} e^{-z / 2} F_{1}[1 / 2+\mu-\kappa ; 1+2 \mu ; z] .
$$

The regularized Whittaker function

$$
\mathcal{M}_{\kappa, \mu}(z)=\frac{M_{\kappa, \mu}(z)}{\Gamma(1+2 \mu)}
$$

is analytic for all values of $\kappa, \mu$, and $z$ real or complex. The second Whittaker function is defined by

$$
\begin{aligned}
W_{\kappa, \mu}(z) & =z^{\mu+1 / 2} e^{-z / 2} U(1 / 2+\mu-\kappa, 1+2 \mu, z) \\
& =\frac{\pi}{\sin (2 \mu \pi)}\left\{\frac{\mathcal{M}_{\kappa,-\mu}(z)}{\Gamma(1 / 2+\mu-\kappa)}-\frac{\mathcal{M}_{\kappa, \mu}(z)}{\Gamma(1 / 2-\mu-\kappa)}\right\}
\end{aligned}
$$

and is analytic for all values of $k, \mu$, and $z$ real or complex, and is even in its second index,

$$
W_{\kappa,-\mu}(z)=W_{\kappa, \mu}(z) .
$$

Whittaker functions $\mathcal{M}_{\kappa, \mu}(z)$ and $W_{\kappa, \mu}(z)$ are the two solutions of the Whittaker differential equation

$$
w_{z z}+\left(-\frac{1}{4}+\frac{\kappa}{z}+\frac{\frac{1}{4}-\mu^{2}}{z^{2}}\right) w=0
$$


with the Wronskian

$$
W_{\kappa, \mu}(z) \mathcal{M}_{\kappa, \mu}^{\prime}(z)-\mathcal{M}_{\kappa, \mu}(z) W_{\kappa, \mu}^{\prime}(z)=\frac{1}{\Gamma(\mu-\kappa+1 / 2)} .
$$

When $\kappa=\mu+n+\frac{1}{2}, n=0,1,2, \ldots$, the Wronskian vanishes and the functions $\mathcal{M}_{\kappa, \mu}(z)$ and $W_{\kappa, \mu}(z)$ become linearly dependent and reduce to generalized Laguerre polynomials (Buchholz 1969, p. 214)

$$
\mathcal{M}_{\mu+n+\frac{1}{2}, \mu}(z)=\frac{n !}{\Gamma(2 \mu+n+1)} e^{-\frac{z}{2}} z^{\mu+\frac{1}{2}} L_{n}^{(2 \mu)}(z),
$$

$$
W_{\mu+n+\frac{1}{2}, \mu}(z)=(-1)^{n} n ! e^{-\frac{z}{2}} z^{\mu+\frac{1}{2}} L_{n}^{(2 \mu)}(z) .
$$

The following integrals with Whittaker functions are used in the proofs of bond and option pricing formulas:

$$
\begin{aligned}
\int_{0}^{x} z^{\alpha-1} e^{-\frac{z}{2}} M_{\kappa, \mu}(z) d z= & \frac{x^{\alpha+\mu+1 / 2}}{\alpha+\mu+1 / 2} \\
& \times{ }_{2} F_{2}[\alpha+\mu+1 / 2,1 / 2+\kappa+\mu ; \alpha+\mu+3 / 2,2 \mu+1 ;-x]
\end{aligned}
$$

for $x>0$ and $\Re(\alpha+\mu+1 / 2)>0$ (Prudnikov, Brychkov, and Marichev 1990, p. 39, equation (1.13.1.1)),

$$
\begin{aligned}
\int_{x}^{\infty} z^{\alpha-1} e^{-\frac{z}{2}} W_{\kappa, \mu}(z) d z= & \frac{\Gamma(\alpha+\mu+1 / 2) \Gamma(\alpha-\mu+1 / 2)}{\Gamma(\alpha-\kappa+1)} \\
& -\frac{x^{\alpha+\mu+1 / 2}}{\alpha+\mu+1 / 2} \frac{\Gamma(-2 \mu)}{\Gamma(1 / 2-\kappa-\mu)} \\
& \times{ }_{2} F_{2}[\alpha+\mu+1 / 2,1 / 2+\kappa+\mu ; \alpha+\mu+3 / 2,2 \mu+1 ;-x] \\
& -\frac{x^{\alpha-\mu+1 / 2}}{\alpha-\mu+1 / 2} \frac{\Gamma(2 \mu)}{\Gamma(1 / 2-\kappa+\mu)} \\
& \times{ }_{2} F_{2}[\alpha-\mu+1 / 2,1 / 2+\kappa-\mu ; \alpha-\mu+3 / 2,-2 \mu+1 ;-x]
\end{aligned}
$$

for $x>0$ (Prudnikov, Brychkov, and Marichev 1990, p. 40, equation (1.13.2.2)),

$$
\int_{0}^{\infty} z^{\alpha-1} e^{-\frac{z}{2}} W_{\kappa, \mu}(z) d z=\frac{\Gamma(\alpha+\mu+1 / 2) \Gamma(\alpha-\mu+1 / 2)}{\Gamma(\alpha-\kappa+1)}
$$

for $\Re(\alpha)>|\Re(\mu)|-1 / 2$ (Prudnikov, Brychkov, and Marichev 1990, p. 256, equation (2.19.3.7)), and indefinite integrals (Prudnikov, Brychkov, and Marichev 1990, pp. 39-40, equations (1.13.1.6) and (1.13.2.6))

$$
\int z^{\kappa-2} e^{-\frac{z}{2}} W_{\kappa, \mu}(z) d z=-z^{\kappa-1} e^{-\frac{z}{2}} W_{\kappa-1, \mu}(z)
$$

$$
\int z^{\kappa-2} e^{-\frac{z}{2}} M_{\kappa, \mu}(z) d z=\frac{1}{\kappa+\mu-1 / 2} z^{\kappa-1} e^{-\frac{z}{2}} M_{\kappa-1, \mu}(z) .
$$


The generalized hypergeometric function is defined by

$$
{ }_{2} F_{2}\left[a_{1}, a_{2} ; b_{1}, b_{2} ; z\right]=\sum_{n=0}^{\infty} \frac{\left(a_{1}\right)_{n}\left(a_{2}\right)_{n}}{\left(b_{1}\right)_{n}\left(b_{2}\right)_{n}} \frac{z^{n}}{n !} .
$$

The regularized function ${ }_{2} F_{2}\left[a_{1}, a_{2} ; b_{1}, b_{2} ; z\right] /\left(\Gamma\left(b_{1}\right) \Gamma\left(b_{2}\right)\right)$ is analytic for all values of $a_{1}, a_{2}, b_{1}, b_{2}$, and $z$ real or complex.

The following integrals with Laguerre polynomials are used in the proofs of pricing formulas:

$$
\int_{0}^{\infty} x^{\alpha-1} e^{-x} L_{n}^{(v)}(x) d x=\frac{(v-\alpha+1)_{n}}{n !} \Gamma(\alpha)
$$

for $\Re(\alpha)>0$ (Prudnikov, Brychkov, and Marichev 1986, p. 463, equation (2.19.3.5)),

$$
\text { (C.15) } \begin{aligned}
\int_{x}^{\infty} z^{\alpha-1} e^{-z} L_{n}^{(v)}(z) d z= & \frac{(v-\alpha+1)_{n}}{n !} \Gamma(\alpha) \\
& -\frac{(v+1)_{n}}{n !} \frac{x^{\alpha}}{\alpha}{ }_{2} F_{2}[v+n+1, \alpha ; v+1, \alpha+1 ;-x]
\end{aligned}
$$

for $x>0$ (Prudnikov, Brychkov, and Marichev 1986, p. 51, equation (1.14.3.7)), and the indefinite integral (Prudnikov, Brychkov, and Marichev 1986, p. 51, equation (1.14.3.9))

$$
\int z^{v+n-1} e^{-z} L_{n}^{(v)}(z) d z=\frac{1}{n} z^{v+n} e^{-z} L_{n-1}^{(v)}(z) .
$$

\section{APPENDIX D: PROOFS}

Proof of Proposition 3.2 (Donati-Martin, Ghomrasni, and Yor 2001). It is classical (Ito and McKean 1974) that, for $s>0$, the resolvent kernel can be taken in the form

$$
G_{s}(x, y)=w_{s}^{-1} \mathfrak{m}(y) \psi_{s}(x \wedge y) \phi_{s}(x \vee y),
$$

where the functions $\psi_{s}(x)$ and $\phi_{s}(x)$ can be characterized as the unique (up to a multiple independent of $x$ ) solutions of the ODE

$$
2 x^{2} u^{\prime \prime}(x)+[2(v+1) x+1] u^{\prime}(x)=\operatorname{su}(x)
$$

by demanding that $\psi_{s}$ is increasing and $\phi_{s}$ is decreasing (Borodin and Salminen 2002, p. 18). These functions have the following limits at zero and infinity (Borodin and Salminen 2002 , pp. 18-19)). At the entrance boundary at zero: $\psi_{s}(0+)>0, \phi_{s}(0+)=\infty$. At the natural boundary at infinity: $\psi_{s}(\infty)=+\infty, \phi_{s}(\infty)=0$. The functions $\psi_{s}(x)$ and $\phi_{s}(x)$ are linearly independent for all $s>0$. Moreover, the Wronskian $w_{s}$ with respect to the scale density $\mathfrak{s}(x)$ defined by

$$
\phi_{s}(x) \psi_{s}^{\prime}(x)-\psi_{s}(x) \phi_{s}^{\prime}(x)=\mathfrak{s}(x) w_{s}
$$

is independent of $x$.

We look for solutions to (D.2) in the form

$$
u(x)=x^{\frac{1-v}{2}} e^{\frac{1}{4 x}} w\left(\frac{1}{2 x}\right)
$$

for some function $w(z)$. Substituting this functional form into equation (D.2), we arrive at the Whittaker equation (C.4) for $w$, where $\kappa=(1-v) / 2$ and $\mu=\mu(s)=\frac{1}{2} \sqrt{2 s+v^{2}}$. For $s>0$, the two linearly independent solutions are $\mathcal{M}_{\kappa, \mu}(z)$ and $W_{\kappa, \mu}(z)$ with the 
Wronskian (C.5). Thus, the solutions $\psi_{s}(x)$ and $\phi_{s}(x)$ of the original problem can be taken in the form

$$
\psi_{s}(x)=x^{\frac{1-v}{2}} e^{\frac{1}{4 x}} W_{\frac{1-v}{2}, \mu(s)}\left(\frac{1}{2 x}\right), \quad \phi_{s}(x)=x^{\frac{1-v}{2}} e^{\frac{1}{4 x}} \mathcal{M}_{\frac{1-v}{2}, \mu(s)}\left(\frac{1}{2 x}\right) .
$$

The boundary properties are verified using the asymptotic properties of the Whittaker functions for $z>0$ and $\mu>0$

$\mathcal{M}_{\kappa, \mu}(z) \sim \frac{1}{\Gamma(1+2 \mu)} z^{\mu+\frac{1}{2}} e^{-\frac{z}{2}} \quad$ and $\quad W_{\kappa, \mu}(z) \sim \frac{\Gamma(2 \mu)}{\Gamma(\mu-\kappa+1 / 2)} z^{-\mu+\frac{1}{2}} e^{-\frac{z}{2}}$ as $z \rightarrow 0$,

(D.6) $\mathcal{M}_{\kappa, \mu}(z) \sim \frac{1}{\Gamma(1 / 2+\mu-\kappa)} z^{-\kappa} e^{\frac{z}{2}} \quad$ and $\quad W_{\kappa, \mu}(z) \sim z^{\kappa} e^{-\frac{z}{2}} \quad$ as $\quad z \rightarrow \infty$.

From (C.5), the Wronskian with respect to the scale density is

$$
w_{s}=\frac{1}{2 \Gamma(\mu(s)+v / 2)}
$$

Substituting (D.4) and (D.7) into (D.1), we arrive at (3.10). The case $x=0$ is obtained in the limit $x \rightarrow 0$, using the asymptotic properties of the Whittaker functions (D.6).

Proof of Proposition 3.3. Following the complex variable approach to spectral expansions (see Titchmarsh 1962), we analytically invert the Laplace transform (3.9) with the resolvent kernel (3.10). Regarded as a function of complex variable $s \in \mathbb{C}$, the resolvent kernel (3.10) has the following singularities. For $v<0$, it has simple poles at

$$
s=s_{n}=-\lambda_{n}, \quad \lambda_{n}=2 n(|v|-n), \quad n=0,1, \ldots,[|\nu| / 2],
$$

(poles of the Gamma function in (3.10) at $\mu\left(-\lambda_{n}\right)+v / 2=-n, n=0,1, \ldots,[|v| / 2]$, where $[x]$ denotes the integer part of $x$ ). The residues at these poles are

(D.9)

$$
\begin{aligned}
& \operatorname{Res}_{s=-\lambda_{n}} G_{s}(x, y) \\
& =(-1)^{n} \frac{2(|v|-2 n)}{n !} e^{\frac{1}{4 x}-\frac{1}{4 y}}\left(\frac{y}{x}\right)^{\frac{\nu-1}{2}} \mathcal{M}_{\frac{1-v}{2},-\frac{v}{2}-n}\left(\frac{1}{2(x \vee y)}\right) W_{\frac{1-v}{2},-\frac{v}{2}-n}\left(\frac{1}{2(x \wedge y)}\right) \\
& =\frac{2(|v|-2 n) n !}{\Gamma(1+|\nu|-n)} e^{-\frac{1}{2 y}}\left(\frac{1}{2 x}\right)^{-n}\left(\frac{1}{2 y}\right)^{1+|v|-n} L_{n}^{(|v|-2 n)}\left(\frac{1}{2 x}\right) L_{n}^{(|v|-2 n)}\left(\frac{1}{2 y}\right) .
\end{aligned}
$$

The second equality follows from the reduction of the Whittaker functions to generalized Laguerre polynomials when the difference between the two indexes is a positive halfinteger (equations (C.6) and (C.7)). Furthermore, for all real $v$, the resolvent has a branch point at $s=-v^{2} / 2$. We place the branch cut from $s=-v^{2} / 2$ to $s \rightarrow-\infty$ on the negative 
real axis. It is convenient to parameterize the branch cut as $\left\{s=-\left(\rho^{2}+v^{2}\right) / 2, \rho \geq 0\right\}$. The jump across the branch cut is

(D.10)

$$
\begin{aligned}
& G_{\frac{1}{2}\left(v^{2}+\rho^{2}\right) e^{i \pi}}(x, y)-G_{\frac{1}{2}\left(v^{2}+\rho^{2}\right) e^{-i \pi}}(x, y) \\
& =-e^{\frac{1}{4 x}-\frac{1}{4 y}}\left(\frac{y}{x}\right)^{\frac{v-1}{2}} W_{\frac{1-v}{2}, \frac{i \rho}{2}}\left(\frac{1}{2(x \wedge y)}\right)\left\{\Gamma\left(\frac{v-i \rho}{2}\right) \mathcal{M}_{\frac{1-v}{2},-\frac{i \rho}{2}}\left(\frac{1}{2(x \vee y)}\right)\right. \\
& \left.-\Gamma\left(\frac{v+i \rho}{2}\right) \mathcal{M}_{\frac{1-v}{2}, \frac{i \rho}{2}}\left(\frac{1}{2(x \vee y)}\right)\right\} \\
& =-\frac{i}{\pi} e^{\frac{1}{4 x}-\frac{1}{4 y}}\left(\frac{y}{x}\right)^{\frac{v-1}{2}} W_{\frac{1-v}{2}, \frac{i \rho}{2}}\left(\frac{1}{2 x}\right) W_{\frac{1-v}{2}, \frac{i \rho}{2}}\left(\frac{1}{2 y}\right)\left|\Gamma\left(\frac{v+i \rho}{2}\right)\right|^{2} \sinh (\pi \rho) .
\end{aligned}
$$

In the first equality we used the fact that $W_{\kappa, \mu}(z)$ is even in its second index. In the second equality we used equation (C.3).

To recover the transition density, we invert the Laplace transform (3.9). The Bromwich complex inversion formula reads for $t>0$

$$
p(t ; x, y)=\frac{1}{2 \pi i} \int_{c-i \infty}^{c+i \infty} e^{s t} G_{s}(x, y) d s,
$$

where the integration is performed along the contour $\operatorname{Re}(s)=c$ for some $c>0$. This integral is calculated by applying the Cauchy Residue Theorem (see Titchmarsh 1962):

(D.11)

$$
\begin{aligned}
p(t ; x, y)= & \mathbf{1}_{\{v<0\}} \sum_{n=0}^{[|v| / 2]} e^{s_{n} t} \operatorname{Res}_{s=s_{n}} G_{s}(x, y) \\
& -\frac{1}{2 \pi i} \int_{0}^{\infty} e^{-\frac{\left(\nu^{2}+\rho^{2}\right) t}{2}}\left\{G_{\frac{1}{2}\left(v^{2}+\rho^{2}\right) e^{i \pi}}(x, y)-G_{\frac{1}{2}\left(\nu^{2}+\rho^{2}\right) e^{-i \pi}}(x, y)\right\} \rho d \rho .
\end{aligned}
$$

Substituting (D.9) and (D.10) in (D.11), we arrive at the spectral representation for the density (3.12).

Since the boundary at zero is entrance, the limit $x \rightarrow 0$ exists and can be explicitly computed using the asymptotics of the Whittaker function (D.6) and Laguerre polynomials

$$
\lim _{z \rightarrow \infty}\left(z^{-n} L_{n}^{(\alpha)}(z)\right)=\frac{(-1)^{n}}{n !} .
$$

Proof of Proposition 3.4. First consider the case $v<2 / p$ (equivalently, $r-q-\sigma^{2} / 2<$ $0)$. Bond payoff $\psi_{\text {bond }}(x)=1$ is such that $\chi_{\psi} \in \mathcal{H}$, and the spectral representation (3.13) is applicable. The integrals in equations (3.14) and (3.15) for $\chi_{\psi}(y)=(y / \beta)^{-\frac{1}{p}}$ are calculated in closed form by reduction to the known integrals (C.10) and (C.14) for Whittaker functions and Laguerre polynomials.

The case $v \geq 2 / p$ (equivalently, $r-q-\sigma^{2} / 2 \geq 0$ ) is more involved. For the bond payoff $\psi_{\text {bond }}(x)=1, \chi_{\psi}(x)=(x / \beta)^{-1 / p} \notin \mathcal{H}$ and, hence, the spectral representation (3.13) cannot be applied. The alternative is to first compute the Laplace transform (3.16) 
with the resolvent kernel (3.10) and then do the Laplace inversion, choosing the contour of integration in the Laplace inversion formula

$$
e^{q T} S^{-1} B(S, T)=E_{x}^{(v)}\left[\left(X_{\tau}^{(v)} / \beta\right)^{-1 / p}\right]=\frac{1}{2 \pi i} \int_{c-i \infty}^{c+i \infty} e^{s \tau} \Phi_{s}^{(v)}(x) d s
$$

to the right of any singularities of $\Phi_{s}^{(v)}(x)$ in the complex $s$-plane. The function $\Phi_{s}^{(v)}(x)$ is calculated in closed form by calculating the integral in (3.16), using the known integrals (C.8) and (C.9). We omit the resulting cumbersome expression. In addition to the singularities inherited from the resolvent kernel $G_{s}(x, y)$ (a branch cut from $s=-v^{2} / 2$ to $-\infty$ on the negative real axis for all real $v$ and poles (D.8) for $v<0)$, for $v>2 / p, \Phi_{s}^{(v)}(x)$ has an additional simple pole at

$$
s=s^{*}=-\lambda^{*}, \quad \lambda^{*}=\frac{2}{p}\left(v-\frac{1}{p}\right)=\frac{4(r-q)}{p^{2} \sigma^{2}}>0
$$

that comes from the factor $1 /(1 / p-v / 2+\mu(s))$. The residue at this pole is

$$
\operatorname{Res}_{s=s^{*}} \Phi_{s}^{(v)}(x)=(x / \beta)^{-\frac{1}{p}} \frac{\Gamma(v-1 / p)}{\Gamma(v-2 / p)} U\left(\frac{1}{p}, \frac{2}{p}-v+1, \frac{1}{2 x}\right),
$$

a strictly positive expression for $v>2 / p$.

The inversion integral (D.12) is calculated by applying the Cauchy Residue Theorem as in the proof of Proposition 3.3 (equation (D.11))

$$
\begin{aligned}
E_{x}^{(v)}\left[\left(X_{\tau}^{(v)} / \beta\right)^{-1 / p}\right]= & \mathbf{1}_{\{v>2 / p\}} e^{s^{*} \tau} \operatorname{Res}_{s=s^{*}} \Phi_{s}^{(v)}(x)+\mathbf{1}_{\{v<0\}} \sum_{n=0}^{[|v| / 2]} e^{s_{n} \tau} \operatorname{Res}_{s=s_{n}} \Phi_{s}^{(v)}(x) \\
& -\frac{1}{2 \pi i} \int_{0}^{\infty} e^{-\frac{\left(v^{2}+\rho^{2}\right) \tau}{2}}\left\{\Phi_{\frac{1}{2}\left(v^{2}+\rho^{2}\right) e^{i \pi}}^{(v)}(x)-\Phi_{\frac{1}{2}\left(v^{2}+\rho^{2}\right) e^{-i \pi}}^{(v)}(x)\right\} \rho d \rho .
\end{aligned}
$$

Computing this expression leads to the result in Proposition 3.4 for the bond price, with the additional pole at $s=s^{*}$ resulting in the additional positive term in the bond pricing formula for $v>2 / p$.

Proof of Proposition 3.5. The put payoff $\psi_{\text {put }}(x)=(K-x)^{+}$is such that $\chi_{\psi} \in \mathcal{H}$ for all $v \in \mathbb{R}$ and, hence, the valuation of the put payoff given no bankruptcy (2.6) follows from the spectral expansion (3.13). The integrals in the expressions for the expansion coefficients (3.14) and (3.15) are calculated in closed form by reduction to the known integrals (C.9) and (C.15). The recovery part (bankruptcy claim) value (2.7) follows from the bond valuation in Proposition 3.4.

Proof of Proposition 4.1. The function $u(S)=\mathbb{P}\left(\lim _{t \rightarrow \infty} S_{t}^{\Delta}=\infty \mid S_{0}=S\right)$ solves the ODE (see Karlin and Taylor 1981, Chapter 12)

$$
\frac{1}{2} \sigma^{2} S^{2} u_{S S}+\left[r-q+\alpha S^{-p}\right] S u_{S}=\alpha S^{-p} u
$$

subject to the boundary conditions

$$
u(0)=0, \quad u(\infty)=1 .
$$

Introducing a new variable $y=1 /(2 x)$, where $x=\left(p \sigma^{2} /(4 \alpha)\right) S^{p}$ as in Section 3 , this ODE reduces to the confluent hypergeometric equation (C.1) with

$$
a=\frac{1}{p}, \quad b=\frac{2}{p}-v+1=\frac{2(q-r)}{p \sigma^{2}}+1+\frac{1}{p} .
$$


The solution satisfying the required boundary conditions is

$$
u(S)=\frac{\Gamma(v-1 / p)}{\Gamma(v-2 / p)} U\left(\frac{1}{p}, \frac{2}{p}-v+1, \frac{1}{2 x}\right) .
$$

The boundary conditions are verified by using the asymptotic properties of the function $U(a, b, x)$

$$
\lim _{x \rightarrow 0} U(a, b, x)=\frac{\Gamma(1-b)}{\Gamma(1+a-b)}
$$

for $b<1$, and

$$
\lim _{x \rightarrow \infty} U(a, b, x)=0
$$

for $a>0$.

\section{REFERENCES}

Abramowitz, M., and I. A. Stegun (1972): Handbook of Mathematical Functions. New York: Dover.

AliLi, L., and J.-C. GRUET (1997): An Explanation of a Generalized Bougerol's Identity in Terms of Hyperbolic Brownian Motion, in Exponential Functionals and Principal Values Related to Brownian Motion, M. Yor, ed. Biblioteca de la Revista Matematica Iberoamericana, Madrid.

Alili, L., H. Matsumoto, and T. Shiraishi (2001): On a Triplet of Exponential Brownian Functionals, Sem. de Prob., xxxv, Springer, Berlin, 396-415.

Andersen, L., and D. Buffum (2003/2004): Calibration and Implementation of Convertible Bond Models, J. Comput. Finance 7(2), 1-34.

AyAche, E., P. Forsyth, and K. Vetzal (2003): The Valuation of Convertible Bonds with Credit Risk, J. Derivatives 11(Fall), 9-29.

Bielecki, T., and M. RutKowski (2002): Credit Risk: Modeling, Valuation and Hedging. Berlin: Springer.

Borodin, A. N., and P. SAlminen (2002): Handbook of Brownian Motion, 2d ed. Boston: Birkhauser.

Buchiolz, H. (1969): The Confluent Hypergeometric Function. Berlin: Springer.

CARR, P., and A. JAVAHERI (2005): The Forward PDE for European Options on Stocks with Fixed Fractional Jumps, Int. J. Theoret. Appl. Finance 8(2), 239-253.

CARR, P., and V. LinETSKY (2005): A Jump-to-Default Extended Constant Elasticity of Variance Model: An Application of Bessel Processes, Working Paper.

Comtet, A. (1987): On the Landau Levels on the Hyperbolic Space, Ann. Phys. 173, 185-209.

Comtet, A., and C. Monthus (1996): Diffusion in a One-Dimensional Random Medium and Hyperbolic Brownian Motion, J. Phys. A 29, 1331-1345.

Comtet, A., C. Monthus, and M. Yor (1998): Exponential Functionals of Brownian Motion and Disordered Systems, J. Appl. Prob. 35, 255-271.

Davis, M., and F. LischKa (2002): Convertible Bonds with Market Risk and Credit Risk, in Applied Probability, R. Chan et al., eds. Studies in Advanced Mathematics. Providence, RI: American Mathematical Society/International Press, 45-58.

Donati-Martin, C., R. Ghomrasni, and M. Yor (2001): On Certain Markov Procsses Attached to Exponential Functionals of Brownian Motion: Applications to Asian Options, Revista Matematica Iberoamericana, 17(1), 179-193. 
Duffie, D., M. Schroder, and C. SkiAdas (1996): Recursive Valuation of Defaultable Securities and the Timing of Resolution of Uncertainty, Ann. Appl. Prob. 6, 1075-1096.

Duffie, D., and K. Singleton (1999): Modeling Term Structures of Defaultable Bonds, Rev. Financial Stud. 12, 653-686.

Duffie, D., and K. Singleton (2003): Credit Risk. Princeton, NJ: Princeton University Press.

Dufresne, D. (1989): Weak Convergence of Random Growth Processes with Applications to Insurance, Insur. Math. Econ. 8, 187-201.

Dufresne, D. (1990): The Distribution of a Perpetuity, with Applications to Risk Theory and Pension Funding, Scand. Actuar. J. 1990, 39-79.

Geman, H., and M. Yor (1992): Some Relations between Bessel Processes, Asian Options and Confluent Hypergeometric Functions, C.R. Acad. Sci., Paris, Ser. I 314, 471-474 (English translation reprinted in Yor, M., 2001, Exponential Functionals of Brownian Motion and Related Processes. Berlin: Springer.).

Geman, H., and M. Yor (1993): Bessel Processes, Asian Options and Perpetuities, Math. Finance 3, 349-375.

Gorovoi, V., and V. LINETSKY (2004): Black's Model of Interest Rates as Options, Eigenfunction Expansions, and Japanese Interest Rates, Math. Finance 14, 49-78.

Grosche, C. (1988): The Path Integral on the Poincaré Upper Half Plane with a Magnetic Field and for the Morse Potential, Ann. Phys. 187, 110-134.

Hull, J., I. Nelken, and A. White (2004): Merton's Model, Credit Risk, and Volatility Skews, Working Paper, University of Toronto.

IkedA, N., and H. Matsumoto (1999): Brownian Motion on the Hyperbolic Plane and Selberg Trace Formula, J. Funct. Anal. 163, 63-110.

IKedA, N., and S. WATANABE (1981): Stochastic Differential Equations and Diffusion Processes. Amsterdam: North-Holland.

Itô, K., and H. McKean, (1974): Diffusion Processes and Their Sample Paths. Berlin: Springer.

JACKWERTh, J. C., and M. RubinsteIn (1996): Recovering Probability Distributions from Option Prices, J. Finance 51, 62-74.

JARrow, R., D. LAndo, and S. Turnbull (1997): A Markov Model for the Term Structure of Credit Risk Spreads, Rev. Financial Stud. 10, 481-523.

Jarrow, R., and S. Turnbull (1995): Pricing Derivatives on Financial Securities Subject to Credit Risk, J. Finance 50(1), 53-85.

JeAnblanc, M., M. Yor, and M. Chesney (2006): Mathematical Methods for Financial Markets. Berlin: Springer.

Karatzas, I., and S. Shreve (1992): Brownian Motion and Stochastic Calculus. New York: Springer-Verlag.

Karlin, S., and H. M. Taylor (1981): A Second Course in Stochastic Processes. San Diego: Academic Press.

Lando, D. (2004): Credit Risk Modeling. Princeton, NJ: Princeton University Press.

LewIS, A. (1998): Applications of Eigenfunction Expansions in Continuous-Time Finance, Math. Finance 8, 349-383.

Linetsky, V. (2004a): Spectral Expansions for Asian (Average Price) Options, Operat. Res. 52(6), 856-867.

Linetsky, V. (2004b): The Spectral Decomposition of the Option Value, Int. J. Theoret. Appl. Finance 7(3), 337-384.

Linetsky, V. (2004c): Lookback Options and Diffusion Hitting Times: A Spectral Expansion Approach, Finance Stochast. 8(3), 373-398. 
Linetsky, V. (2004d): The Spectral Representation of Bessel Processes with Constant Drift: Applications in Queueing and Finance, J. Appl. Prob. 41(2), 327-344.

Linetsky, V. (2006): Spectral Methods in Derivatives Pricing, in Handbook of Financial Engineering, J. R. Birge and V. Linetsky, eds. Amsterdam: Elsevier.

Madan, D., and H. Unal (1998): Pricing the Risk of Default, Rev. Deriv. Res. 2, 121-160.

McKean, H. (1956): Elementary Solutions for Certain Parabolic Partial Differential Equations, Transactions of the American Mathematical Society 82, 519-548.

Morse, P. M. (1929): Diatomic Molecules According to the Wave Mechanics, II: Vibrational Levels, Phys. Rev. 34, 57-64.

Muromachi, Y. (1999): The Growing Recognition of Credit Risk in Corporate and Financial Bond Markets, Working Paper, NLI Research Institute.

PeskiR, G. (2004): On the Fundamental Solution of the Kolmogorov-Shiryaev Equation, Research Report No. 438, Aarhus.

Prudnikov, A. P., Y. A. Brychkov, and O. I. Marichev (1986): Integrals and Series, Vol. 2. New York: Gordon and Breach.

Prudnikov, A. P., Y. A. Brychkov, and O. I. Marichev (1990): Integrals and Series, Vol. 3. New York: Gordon and Breach.

Revuz, D., and M. Yor (1999): Continuous Martingales and Brownian Motion, 3rd edn. Berlin: Springer.

Rubinstein, M. (1994): Implied Binomial Trees, J. Finance 49, 771-818.

Schoönbucher, P. J. (2003): Credit Derivatives Pricing Models. New York: Wiley.

Shiryaev, A. N. (1961): The Problem of the Most Rapid Detection of a Disturbance in a Stationary Process, Soviet Mathematics Doklady 2, 795-799.

Slater, L. J. (1960): Confluent Hypergeometric Functions. Cambridge, UK: Cambridge University Press.

Takahashi, A., T. Kobayashi, and N. Nakagawa (2001): Pricing Convertible Bonds with Default Risk, J. Fixed Income 11, 20-29.

Titchmarsh, E. C. (1962): Eigenfunction Expansions Associated with Second-Order Differential Equations. Oxford: Clarendon.

Wong, E. (1964): The Construction of a Class of Stationary Markoff Processes, in Sixteenth Symposium in Applied Mathematics-Stochastic Processes in Mathematical Physics and Engineering, R. Bellman, ed. Providence, RI: American Mathematical Society, 264-276.

Yor, M. (2001): Exponential Functionals of Brownian Motion and Related Processes. Berlin: Springer. 